Pacific

Journal of

Mathematics

\title{
A COMBINATORIAL CHARACTERIZATION OF TIGHT FUSION FRAMES
}

MARCIN BOWNiK, KuRT LUOTO AND EDWARD RICHMOND 


\title{
A COMBINATORIAL CHARACTERIZATION OF TIGHT FUSION FRAMES
}

\author{
MARCIN BOWNIK, KURT LUOTO AND EDWARD RICHMOND
}

\begin{abstract}
In this paper we give a combinatorial characterization of tight fusion frame (TFF) sequences using Littlewood-Richardson skew tableaux. The equal rank case has been solved recently by Casazza, Fickus, Mixon, Wang, and Zhou. Our characterization does not have this limitation. We also develop some methods for generating TFF sequences. The basic technique is a majorization principle for TFF sequences combined with spatial and Naimark dualities. We use these methods and our characterization to give necessary and sufficient conditions which are satisfied by the first three highest ranks. We also give a combinatorial interpretation of spatial and Naimark dualities in terms of Littlewood-Richardson coefficients. We exhibit four classes of TFF sequences which have unique maximal elements with respect to majorization partial order. Finally, we give several examples illustrating our techniques including an example of tight fusion frame which can not be constructed by the existing spectral tetris techniques. We end the paper by giving a complete list of maximal TFF sequences in dimensions $\leq 9$.
\end{abstract}

\section{Introduction}

Fusion frames were introduced in [Casazza and Kutyniok 2004] (under the name frames of subspaces) and in [Casazza et al. 2008]. A fusion frame for $\mathbb{R}^{N}$ is a finite collection of subspaces $\left\{W_{i}\right\}_{i=1}^{K}$ in $\mathbb{R}^{N}$ such that there exist constants $0<\alpha \leq \alpha^{\prime}<\infty$ satisfying

$$
\alpha\|x\|^{2} \leq \sum_{i=1}^{K}\left\|P_{i} x\right\|^{2} \leq \alpha^{\prime}\|x\|^{2} \quad \text { for all } x \in \mathbb{R}^{N},
$$

where $P_{i}$ is the orthogonal projection onto $W_{i}$. Equivalently, $\left\{W_{i}\right\}_{i=1}^{K}$ is a fusion frame if and only if

$$
\alpha \mathbf{I} \leq \sum_{i=1}^{K} P_{i} \leq \alpha^{\prime} \mathbf{I}
$$

MSC2010: primary 05E05, 15A57, 42C15; secondary 14N15, 14M15.

Keywords: tight fusion frame, majorization, orthogonal projection, partition, Schur function,

Littlewood-Richardson coefficient, Schubert calculus, symmetric functions. 
where $\mathbf{I}$ is the identity on $\mathbb{R}^{N}$. The constants $\alpha$ and $\alpha^{\prime}$ are called fusion frame bounds. An important class of fusion frames are tight fusion frames (TFF), for which $\alpha=\alpha^{\prime}$ and hence $\sum_{i=1}^{K} P_{i}=\alpha \mathbf{I}$. We note that the definition of fusion frames given in [Casazza and Kutyniok 2004; Casazza et al. 2008] applies to closed subspaces in any Hilbert space together with a collection of weights associated to each subspace $W_{i}$. Since the scope of this paper is limited to nonweighted finite dimensional TFF, the definition of a fusion frame is only presented for this case.

Fusion frames have been a very active area of research in the frame theory [Casazza and Kutyniok 2013]. A lot of effort was devoted into developing the basic properties and constructing fusion frames with desired properties. In particular, the construction and existence of sparse tight fusion frames was studied in [Calderbank et al. 2011]. Fusion frame potentials have been studied in [Casazza and Fickus 2009] and [Massey et al. 2010]. Applications of fusion frames include sensor networks [Casazza et al. 2008], coding theory [Bodmann 2007; Kutyniok et al. 2009], compressed sensing [Boufounos et al. 2011], and filter banks [Chebira et al. 2011]. In this paper we consider a problem of classifying TFF sequences.

Problem 1.1. Given $N \in \mathbb{N}$, characterize sequences $\left(L_{1}, \ldots, L_{K}\right)$ for which there exists a tight fusion frame $\left\{W_{i}\right\}_{i=1}^{K}$ with $\operatorname{dim} W_{i}=L_{i}$ in $N$ dimensional space. Equivalently, given $\alpha>1$ such that $\alpha N \in \mathbb{N}$, characterize sequences $\left(L_{1}, \ldots, L_{K}\right)$ such that $\alpha \mathbf{I}$ can be decomposed as a sum of projections $P_{1}+\cdots+P_{K}$ with $\operatorname{rank} P_{i}=L_{i}, i=1, \ldots, K$.

Casazza, Fickus, Mixon, Wang, and Zhou [Casazza et al. 2011] have recently achieved significant progress in this direction by solving the equal rank case. That is, the authors have classified all triples $(K, L, N)$ such that there exists a tight fusion frame consisting of $K$ subspaces $\left\{W_{i}\right\}_{i=1}^{K}$ with the same dimension $\operatorname{dim} W_{i}=L$ in $\mathbb{R}^{N}$. The answer is highly nontrivial in the most interesting case when $L$ does not divide $N$ and $2 L<N$. The authors show that a necessary condition for such sequences $(K, L, N)$ is that $K \geq\lceil N / L\rceil+1$, whereas a sufficient condition is $K \geq\lceil N / L\rceil+2$. In a gray area, where $K=\lceil N / L\rceil+1$, the authors have devised a reduction procedure which replaces the original sequence by another one with the equivalent TFF property (existence or nonexistence). Then, it is shown that after a finite number of steps the original sequence $(K, L, N)$ is reduced to one for which either the necessary condition fails or the sufficient condition holds. However, the results [Casazza et al. 2011] do not say much about a more general problem of classifying TFF sequences with unequal ranks. In this paper we answer Problem 1.1 by giving a combinatorial characterization of TFF sequences using Littlewood-Richardson skew tableaux.

While the concept of fusion frames is relatively new, the problem of representing an operator as a sum of orthogonal projections has been studied for a long time in 
the operator theory. The first fundamental result of this kind belongs to Fillmore [1969] who characterized finite rank operators which are finite sums of projections; see Theorem 3.1. Fong and Murphy [1985] characterized operators which are positive combinations of projections. Analogous results were recently investigated for C-* algebras and von Neumann algebras; see [Halpern et al. 2013; Kaftal et al. 2011]. However, the most relevant results for us are due to Kruglyak, Rabanovich, and Samorlenko [Kruglyak et al. 2002; 2003] who characterized the set of all $(\alpha, N)$ such that $\alpha \mathbf{I}$ is the sum of $K$ orthogonal projections. In other words, their main result (Theorem 7 of the latter reference) gives a minimal length $K$ of a TFF sequence in $\mathbb{R}^{N}$ with the frame bound $\alpha$. However, their results do not say anything about the ranks of projections which is a focus of this paper.

In the finite dimensional setting the existence of TFF sequences is intimately related to Horn's problem [Horn 1962] which has been solved by Klyachko [1998], and Knutson and Tao [Knutson and Tao 1999; Knutson et al. 2004], for a survey see [Fulton 2000; Knutson and Tao 2001]. Problem 1.1 can be thought of as a very special kind of Horn's problem where hermitian matrices have only two eigenvalues: 0 and 1, and their sum has only one eigenvalue $\alpha$. Using Klyachko's result [1998] we show that the existence of TFF sequence $\left(L_{1}, \ldots, L_{K}\right)$ is equivalent to the nonvanishing of a certain Littlewood-Richardson coefficient; see Theorem 4.3. In turn, the latter condition is equivalent to the existence of a matrix satisfying some computationally explicit properties such as: constant row and column sums, and row and column sum dominance; see Corollary 4.4. Our combinatorial characterization enables us to deduce several properties that TFF sequences must satisfy. In addition, it enables us to give an explicit construction procedure of a tight fusion frame corresponding to a given TFF sequence; see Example 7.2.

A fundamental technique of our paper is a majorization principle involving the majorization partial order $\preccurlyeq$ as in the Schur-Horn theorem [Antezana et al. 2007; Kaftal and Weiss 2010], which is also known as the dominance order in algebraic combinatorics [Fulton 1997]. In Section 2 we show that a sequence majorized by a TFF sequence is also a TFF sequence. We also establish the spatial and Naimark dualities for general TFF sequences extending the equal rank results in [Casazza et al. 2011]. In Section 3 we find necessary and sufficient conditions on the first three largest ranks of projections using Filmore's theorem [1969] and a description of possible spectra of a sum of two projections; see Lemma 3.2. The latter result might be of independent interest since its proof uses honeycomb models developed by Knutson and Tao [1999; 2001]. In the same section we also exhibit classes of TFF sequences which have only one maximal element. These include not only the expected case of integer $\alpha$, but also half-integer scenario, and the corresponding conjugate $\alpha$ 's via the Naimark duality. In Section 4 we prove our main characterization result of TFF sequences using Littlewood-Richardson skew 
tableaux. In addition to illustrating it on specific examples, in Section 5 we give a complete proof of Theorem 3.3 using the combinatorics of the Schur functions. This leads to a partial characterization of TFF sequences which are of the hook type, i.e., sequences ending in repeated 1's. In Section 6 we show that the spatial and Naimark dualities manifest themselves as identities for the corresponding Littlewood-Richardson coefficients. Finally, in Section 7 we give several examples of existence of tight fusion frames using skew Littlewood-Richardson tableaux. In particular, we give an explicit construction of TFF corresponding to the sequence $(4,2,2,2,1)$ in dimension $N=6$. This example is remarkable for two reasons. It is the first TFF sequence which is missed by brute force generation involving recursive spatial and Naimark dualities. Furthermore, this example can not be constructed by the existing spectral tetris construction [Calderbank et al. 2011; Casazza et al. 2012], which is an algorithmic method of constructing sparse fusion frames utilized in the equal rank characterization [Casazza et al. 2011]. We end the paper by giving a complete list of maximal TFF sequences for $\alpha \leq 2$ in dimensions $N \leq 9$.

\section{Basic majorization and duality results}

Definition 2.1. Fix a positive integer $N$. Let $L_{1} \geq L_{2} \geq \cdots \geq L_{K}>0$ be a weakly decreasing sequence of positive integers. Such sequence is also known as a partition in number theory [Andrews 1976] and algebraic combinatorics [Fulton 1997]. We say that $\left(L_{1}, L_{2}, \ldots, L_{K}\right)$ is a tight fusion frame (TFF) sequence if there exists orthogonal projections $P_{1}, \ldots, P_{K}$ such that

$$
\alpha \mathbf{I}=\sum_{i=1}^{K} P_{i}, \quad \text { and } \quad \operatorname{rank}_{i}=L_{i},
$$

where $\alpha \in \mathbb{R}$ and $\mathbf{I}$ is the identity on $\mathbb{R}^{N}$. A trace argument shows that $\alpha=$ $\sum_{i=1}^{K} L_{i} / N \geq 1$. Given $\alpha \geq 1$ such that $\alpha N \in \mathbb{N}$, we define $\operatorname{TFF}(\alpha, N)$ to be the set of all TFF sequences in $\mathbb{R}^{N}$ with the frame bound $\alpha$.

Majorization. The following definition comes from the majorization theory of the Schur-Horn theorem; see [Kaftal and Weiss 2010]. In algebraic combinatorics the majorization partial order on partitions is known as the dominance order; see [Fulton 1997].

Definition 2.2. Suppose that $\mathbf{L}=\left(L_{1}, L_{2}, \ldots, L_{K}\right)$ and $\mathbf{L}^{\prime}=\left(L_{1}^{\prime}, L_{2}^{\prime}, \ldots, L_{K^{\prime}}^{\prime}\right)$ be two weakly decreasing sequences of nonnegative integers. We say that $\mathbf{L}^{\prime}$ majorizes $\mathbf{L}$, and write $\mathbf{L} \preccurlyeq \mathbf{L}^{\prime}$ if for all $k \leq \min \left(K, K^{\prime}\right)$,

$$
\sum_{i=1}^{K} L_{i}=\sum_{i=1}^{K^{\prime}} L_{i}^{\prime} \quad \text { and } \quad \sum_{i=1}^{k} L_{i} \leq \sum_{i=1}^{k} L_{i}^{\prime} .
$$


Observe that appending zeros at the tails of sequences $\mathbf{L}, \mathbf{L}^{\prime}$ does not affect majorization relation. Moreover, for sequences with only positive terms, the majorization $\mathbf{L} \preccurlyeq \mathbf{L}^{\prime}$ forces $K \geq K^{\prime}$.

The majorization principle for TFF sequences takes the following form.

Theorem 2.3. Let $\mathbf{L}$ and $\mathbf{L}^{\prime}$ be two weakly decreasing sequences of positive integers such that $\mathbf{L} \preccurlyeq \mathbf{L}^{\prime}$. Then, $\mathbf{L}^{\prime} \in \operatorname{TFF}(\alpha, N)$ implies that $\mathbf{L} \in \operatorname{TFF}(\alpha, N)$.

In the proof of Theorem 2.3 we use the following elementary result on a sum of two projections.

Lemma 2.4. Fix positive integers $p>q \geq 0$. Let $P$ and $Q$ be two orthogonal projection of ranks $p$ and $q$, respectively. Then, there exist orthogonal projections $P^{\prime}$ and $Q^{\prime}$ of ranks $p-1$ and $q+1$, respectively, such that $P+Q=P^{\prime}+Q^{\prime}$.

Proof. Assume we have two projections $P$ and $Q$ with ranks $p>q$ that act on an $N$ dimensional vector space $V$. Then, we can decompose $V$ into the eigenspaces of $P$ and $Q$ such that

$$
V=V_{P} \oplus V_{P}^{\perp}, \quad V=V_{Q} \oplus V_{Q}^{\perp},
$$

where $V_{P}$ and $V_{P}^{\perp}$ denote the 1-eigenspace and 0-eigenspace, respectively. Since $p>q$, we have that $p+(N-q)>N$ and hence $\operatorname{dim}\left(V_{P} \cap V_{Q}^{\perp}\right)>0$. Choose a nonzero vector in $V_{P} \cap V_{Q}^{\perp}$ and let $R$ denote the corresponding rank 1 projection. Then, we can decompose $P=\bar{P}+R$, where $\bar{P}$ is a rank $p-1$ projection. Moreover, $Q+R$ is a projection of rank $q+1$. Thus, $P+Q=\bar{P}+(Q+R)$, which completes the proof of the lemma.

Proof of Theorem 2.3. Since $\mathbf{L} \preccurlyeq \mathbf{L}^{\prime}$ we can find a sequence of partitions $\mathbf{L}=$ $\mathbf{L}^{0} \preccurlyeq \mathbf{L}^{1} \preccurlyeq \cdots \preccurlyeq \mathbf{L}^{n}=\mathbf{L}^{\prime}$ such that any two consecutive partitions $\mathbf{L}^{j-1}$ and $\mathbf{L}^{j}$, $j=1, \ldots, n$, differ at exactly two positions by \pm 1 . That is, for each $j=1, \ldots, n$, there exist two positions $m<m^{\prime} \in \mathbb{N}$ such that

$$
\begin{aligned}
\mathbf{L}^{j-1} & =\left(*, \ldots, *, \tilde{L}_{m} \quad, *, \ldots, *, \tilde{L}_{m^{\prime}} \quad, *, \ldots, *\right), \\
\mathbf{L}^{j} & =\left(*, \ldots, *, \tilde{L}_{m}+1, *, \ldots, *, \tilde{L}_{m^{\prime}}-1, *, \ldots, *\right),
\end{aligned}
$$

where the remaining values, denoted by $*$, are the same. Such partitions $\mathbf{L}^{j}$ can be easily constructed by the following recursive procedure.

Given the initial partitions $\mathbf{L}$ and $\mathbf{L}^{\prime}$ we append extra zeros to $\mathbf{L}^{\prime}$ so that $\mathbf{L}$ and $\mathbf{L}^{\prime}$ have the same length. Define $m$ to be the first position such that initial subsequences $\left(L_{1}, \ldots, L_{m}\right)$ and $\left(L_{1}^{\prime}, \ldots, L_{m}^{\prime}\right)$ are not the same. Likewise, $m^{\prime}$ is the last position such that the ending subsequences $\left(L_{m^{\prime}}, \ldots\right)$ and $\left(L_{m^{\prime}}^{\prime}, \ldots\right)$ are not the same. Define $\mathbf{L}^{1}$ from $\mathbf{L}$ by replacing $L_{m} \rightarrow L_{m}+1$ and $L_{m^{\prime}} \rightarrow L_{m^{\prime}}-1$. It is not difficult to see that $\mathbf{L}^{1}$ forms a weakly decreasing sequence and $\mathbf{L}=\mathbf{L}_{0} \preccurlyeq \mathbf{L}^{1} \preccurlyeq \mathbf{L}^{\prime}$. 
Repeating this procedure recursively we define a sequence $\mathbf{L}^{1} \preccurlyeq \mathbf{L}^{2} \preccurlyeq \cdots \preccurlyeq \mathbf{L}^{\prime}$. After a finite number of steps we must arrive at $\mathbf{L}^{n}=\mathbf{L}^{\prime}$.

Observe that the ranks in (2-2) satisfy $\tilde{L}_{m} \geq \tilde{L}_{m^{\prime}}$. By Lemma 2.4 applied to two projections with ranks $p=\tilde{L}_{m}+1>q=\tilde{L}_{m^{\prime}}-1 \geq 0$, if $\mathbf{L}^{j} \in \operatorname{TFF}(\alpha, N)$, then $\mathbf{L}^{j-1} \in \operatorname{TFF}(\alpha, N)$. Therefore, repeated application of Lemma 2.4 proves Theorem 2.3.

We remark that the above proof does not use the tightness assumption in any way. Consequently, Theorem 2.3 holds for general (not necessarily tight) fusion frames with a prescribed frame operator.

Dualities. In this subsection we shall establish two dualities for TFF sequences. The first duality involves taking orthogonal projections of the same ambient space and is a straightforward generalization of [Casazza et al. 2011, Theorem 6].

Theorem 2.5. Suppose that $\left(L_{1}, L_{2}, \ldots, L_{K}\right) \in \operatorname{TFF}(\alpha, N)$. Then, $\left(N-L_{K}, N-\right.$ $\left.L_{K-1}, \ldots, N-L_{1}\right) \in \operatorname{TFF}(K-\alpha, N)$.

Proof. Let $P_{1}, \ldots, P_{K}$ be the orthogonal projections with rank $P_{i}=L_{i}$ such that $\sum_{i=1}^{K} P_{i}=\alpha \mathbf{I}$. Clearly, $\sum_{i=1}^{K}\left(\mathbf{I}-P_{i}\right)=(K-\alpha) \mathbf{I}$ and $\operatorname{rank}\left(\mathbf{I}-P_{i}\right)=N-L_{i}$.

The second result relies on taking more subtle orthogonal complements based on a dilation theorem for tight frames with bound 1, also known as Parseval frames. It is known that every Parseval frame can be obtained as a projection of an orthogonal basis of some higher dimensional space. The complementary projection gives rise to another Parseval frame, which is often called the Naimark complement of the original frame. This leads to the following result

Theorem 2.6. Suppose that $\left(L_{1}, L_{2}, \ldots, L_{K}\right) \in \operatorname{TFF}(\alpha, N)$. Then, the same sequence $\left(L_{1}, L_{2}, \ldots, L_{K}\right) \in \operatorname{TFF}(\tilde{\alpha}, \tilde{N})$, where the dimension $\tilde{N}=\left(\sum_{i=1}^{K} L_{i}-N\right)$ and the frame bound $\tilde{\alpha}=\alpha /(\alpha-1)=\alpha N / \tilde{N}$.

Proof. For each $k=0, \ldots, K$, define $\sigma_{k}=\sum_{i=1}^{k} L_{i}$ with the convention that $\sigma_{0}=0$. Our assumption implies that there exists a tight frame $\left\{v_{j}\right\}_{j=1}^{\sigma_{K}}$ in $\mathbb{R}^{N}$ such that for each $k=1, \ldots, K$, the subcollection $\left\{v_{j}\right\}_{j=1+\sigma_{k-1}}^{\sigma_{k}}$ is an orthonormal sequence which spans the $L_{k}$ dimensional space $W_{k}$ from the definition of a TFF. Treating $v_{1}, \ldots, v_{\sigma_{K}}$ as column vectors we obtain an $N \times \sigma_{K}$ matrix $U$ with orthogonal rows each of norm $\sqrt{\alpha}=\sqrt{\sigma_{K} / N}$. This is due to the fact that $\left\{v_{j}\right\}_{j=1}^{\sigma_{K}}$ is a tight frame with constant $\alpha$.

Let $\widetilde{U}$ be an extension of $U$ to a $\sigma_{K} \times \sigma_{K}$ matrix with all orthogonal rows of norm $\sqrt{\alpha}$. In other words, $(1 / \sqrt{\alpha}) \widetilde{U}$ is a unitary extension of $(1 / \sqrt{\alpha}) U$ which has orthonormal rows. Let $\left\{w_{j}\right\}_{j=1}^{\sigma_{K}}$ be the column vectors constituting the $\left(\sigma_{K}-N\right) \times \sigma_{K}$ submatrix of the bottom rows of $\widetilde{U}$. Since $(1 / \sqrt{\alpha}) \widetilde{U}$ is an orthogonal matrix we have

$$
\left\langle v_{j}, v_{j^{\prime}}\right\rangle+\left\langle w_{j}, w_{j^{\prime}}\right\rangle=\alpha \delta_{j, j^{\prime}} \quad \text { for all } j, j^{\prime}=1, \ldots, \sigma_{K} .
$$


By the block orthogonality of vectors $v_{j}$ we have that for each block $k=1, \ldots, K$,

$$
\left\langle w_{j}, w_{j^{\prime}}\right\rangle=(\alpha-1) \delta_{j, j^{\prime}} \quad \text { for all } j, j^{\prime}=1+\sigma_{k-1}, \ldots, \sigma_{k} .
$$

This means that the vectors $\left\{w_{j}\right\}_{j=1+\sigma_{k-1}}^{\sigma_{k}}$ form an orthogonal set which span some $L_{k}$ dimensional space $\widetilde{W}_{k}$. Moreover, $\left\{w_{j}\right\}_{j=1}^{\sigma_{K}}$ is a tight frame with a constant $\alpha$ for $\left(\sigma_{K}-N\right)$ dimensional space. Consequently, unit norm vectors $\left\{(1 / \sqrt{\alpha-1}) w_{j}\right\}_{j=1}^{\sigma_{K}}$, which are block orthonormal, form a tight frame with a constant $\alpha /(\alpha-1)$. This leads to the decomposition $\widetilde{P}_{1}+\cdots+\widetilde{P}_{K}=\alpha /(\alpha-1) \mathbf{I}$, where $\widetilde{P}_{k}$ is an orthogonal projection onto $\widetilde{W}_{k}$.

As an immediate corollary of Theorem 2.6, we can reduce the study of TFF sequences to the case when $1<\alpha<2$; the case $\alpha=2$ does not cause any difficulties as we will see later.

Corollary 2.7. If $\alpha>1$ is such that $\alpha N \in \mathbb{N}$, then $\operatorname{TFF}(\alpha, N)=\operatorname{TFF}(\tilde{\alpha}, \tilde{N})$, where $1 / \alpha+1 / \tilde{\alpha}=1$ and $\tilde{N}=N(\alpha-1)$.

Observe that if there exists a TFF sequence with parameters $(\alpha, N)$, then by computing traces we necessarily have that $\alpha N \in \mathbb{N}$. Hence, without loss of generality we shall always make this assumption.

\section{Estimates on first 3 ranks}

In this section we find necessary and sufficient conditions on the first three largest ranks of TFF projections. Our analysis is based on two fundamental results. Theorem 3.1 is due to Fillmore. Lemma 3.2 describes the spectral properties of the sum of two projections, and it can be thought of as a generalization of Lemma 2.4.

Theorem 3.1 [Fillmore 1969, Theorem 1]. A nonnegative definite hermitian matrix $S$ is a sum of projections if and only if

$$
\operatorname{trace}(S) \in \mathbb{N}_{0} \quad \text { and } \quad \operatorname{trace}(S) \geq \operatorname{rank}(S) .
$$

Lemma 3.2. Let $P, Q$ be two orthogonal projections on an $N$ dimensional vector space $V$ with ranks $p, q$, respectively. For any $\lambda \in \mathbb{R}$, let $m(\lambda)$ be the multiplicity of $\lambda$ as an eigenvalue of $P+Q$. Then, the following are true:

(i) $m(\lambda)>0 \Longrightarrow \lambda \in[0,2]$,

(ii) $\sum_{\lambda \in[0,2]} m(\lambda)=N$,

(iii) $m(1) \geq|p-q|$,

(iv) $\lambda \in(0,2) \Longrightarrow m(\lambda)=m(2-\lambda)$,

(v) $m(0)-m(2)=N-p-q$. 
Conversely, if $0 \leq p, q \leq N$ and $m: \mathbb{R} \rightarrow \mathbb{N}_{0}$ satisfies (i)-(v), then there exists orthogonal projections $P, Q$ of ranks $p, q$, such that $m$ is a multiplicity function of $P+Q$.

Proof. Since $P, Q$ are hermitian, we can decompose $V$ as a direct sum of eigenspaces

$$
V=V_{P} \oplus V_{P}^{\perp}=V_{Q} \oplus V_{Q}^{\perp}
$$

where $V_{P}$ denotes the 1-eigenspace and $V_{P}^{\perp}$ the 0 eigenspace of $P$. Thus, $p=$ $\operatorname{dim}\left(V_{P}\right)$ and $q=\operatorname{dim}\left(V_{Q}\right)$. Parts (i)-(iii) follow by basic linear algebra.

To prove part (iv) we define $f_{\lambda}: V \rightarrow V$ by

$$
f_{\lambda}(v):=v_{P}+\left(\frac{\lambda}{\lambda-2}\right) v_{P}^{\prime},
$$

where $v=v_{P}+v_{P}^{\prime}$ is induced by the orthogonal decomposition $V=V_{P} \oplus V_{P}^{\perp}$ and $\lambda \in(0,2)$. Since $f_{\lambda}$ is an invertible and linear map, it suffices to show that if $(P+Q) v=\lambda v$, then $(P+Q) f_{\lambda}(v)=(2-\lambda) f_{\lambda}(v)$. Write

$$
v_{P}=x_{Q}+x_{Q}^{\prime} \quad \text { and } \quad v_{P}^{\prime}=y_{Q}+y_{Q}^{\prime}
$$

according to the decomposition $V=V_{Q} \oplus V_{Q}^{\perp}$. Then,

$$
(P+Q) v=v_{P}+x_{Q}+y_{Q}=2 x_{Q}+y_{Q}+x_{Q}^{\prime}=\lambda\left(x_{Q}+x_{Q}^{\prime}+y_{Q}+y_{Q}^{\prime}\right)
$$

and hence

$$
(2-\lambda) x_{Q}+(1-\lambda) y_{Q}=(\lambda-1) x_{Q}^{\prime}+\lambda y_{Q}^{\prime} .
$$

This implies that

$$
(2-\lambda) x_{Q}=(\lambda-1) y_{Q} \quad \text { and } \quad(1-\lambda) x_{Q}^{\prime}=\lambda y_{Q}^{\prime}
$$

since $V_{Q} \cap V_{Q}^{\perp}=\{0\}$.

By (3-2), we have

$$
\begin{aligned}
(P+Q) f_{\lambda}(v) & =2 x_{Q}+x_{Q}^{\prime}+\left(\frac{\lambda}{\lambda-2}\right) y_{Q} \\
& =(2-\lambda) v_{P}+\lambda x_{Q}+(\lambda-1) x_{Q}^{\prime}+\left(\frac{\lambda}{\lambda-2}\right) y_{Q} \\
& =(2-\lambda) v_{P}+\left(\frac{\lambda(1-\lambda)}{\lambda-2}\right) y_{Q}-\lambda y_{Q}^{\prime}+\left(\frac{\lambda}{\lambda-2}\right) y_{Q} \\
& =(2-\lambda) v_{P}-\lambda y_{Q}-\lambda y_{Q}^{\prime} \\
& =(2-\lambda)\left(v_{P}+\left(\frac{\lambda}{\lambda-2}\right) v_{P}^{\prime}\right)=(2-\lambda) f_{\lambda}(v) .
\end{aligned}
$$

This proves part (iv). To prove part (v), we consider the projection map

$$
g: V \rightarrow V_{P}+V_{Q}
$$


where $V_{P}+V_{Q}$ denotes the span of vectors in $V_{P}, V_{Q}$. We have

$$
\operatorname{dim}\left(V_{P}+V_{Q}\right)=\operatorname{dim}\left(V_{P}\right)+\operatorname{dim}\left(V_{Q}\right)-m(2)=p+q-m(2) .
$$

But,

$$
\operatorname{dim}\left(V_{P}+V_{Q}\right)=N-\operatorname{dim}(\operatorname{ker} g)=N-m(0) .
$$

This shows that the properties (i)-(v) are necessary.

A quick way to see the converse direction is to utilize the honeycomb model of Knutson and Tao [1999; 2001]. The honeycombs corresponding to triples $(P, Q,-(P+Q))$, where $p>q$ can be represented by one of the following diagrams. In the case $p=q$ the line corresponding the eigenvalue -1 of $-(P+Q)$ might not be present. We leave the details to the reader. This involves finding multiplicities of unlabeled line segments to satisfy the "zero-tension" property.

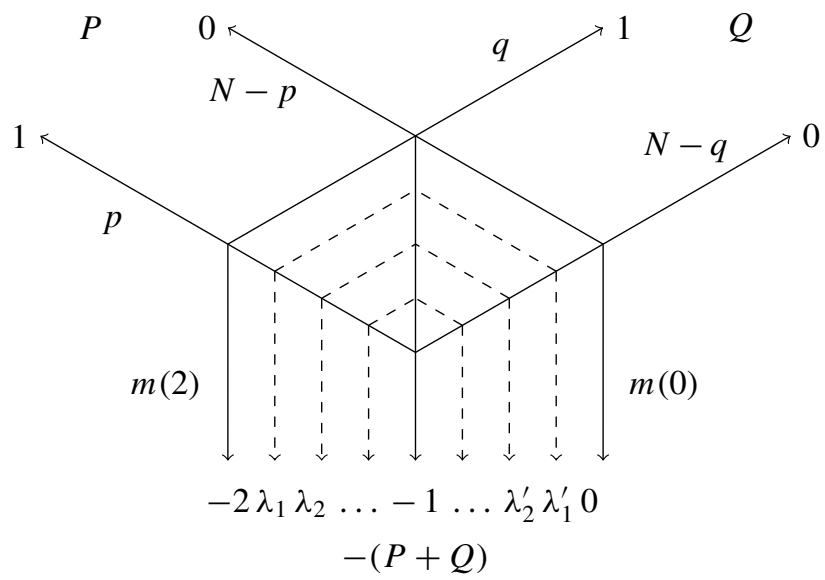

Figure 1. Honeycomb with $m(2)>0, m(0)>0$ and $\lambda_{i}^{\prime}:=-2-\lambda_{i}$.

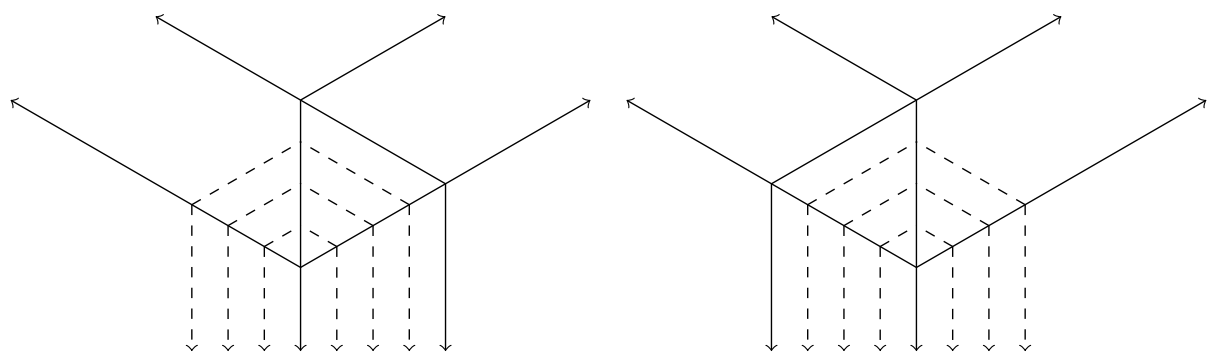

Figure 2. Honeycombs with $m(2)=0$ and $m(0)=0$, respectively. 
Using Theorem 3.1 and Lemma 3.2, our goal is to find necessary and sufficient conditions on the first three largest ranks of projections in a TFF.

Theorem 3.3. Suppose that $1<\alpha<2$ and $\left(L_{1} \geq L_{2} \geq \cdots \geq L_{K}\right) \in \operatorname{TFF}(\alpha, N)$. Then, we have the following necessary conditions:

$$
\begin{aligned}
L_{1} & \leq(\alpha-1) N, \\
L_{1}+L_{2} & \leq N, \\
L_{1}+L_{2}+L_{3} & \leq \begin{cases}N, & \alpha<3 / 2, \\
2(\alpha-1) N, & \alpha>3 / 2 .\end{cases}
\end{aligned}
$$

Conversely, if $L_{1} \geq L_{2} \geq L_{3}$ satisfy (3-3), (3-4), and (3-5), then there exists $\mathbf{L} \in \operatorname{TFF}(\alpha, N)$ which starts with the sequence $\left(L_{1}, L_{2}, L_{3}\right)$.

Proof. Suppose $\alpha \mathbf{I}$ is written as in (2-1). Then, $S=\alpha \mathbf{I}-P_{1}$ is an operator with 2 eigenvalues: $\alpha$ with multiplicity $N-L_{1}$ and $(\alpha-1)$ with multiplicity $L_{1}$. By Theorem 3.1 we must have

$$
\alpha N-L_{1} \geq N .
$$

Solving this for $L_{1}$ yields (3-3).

By Lemma 3.2 the sum $P_{1}+P_{2}$ has eigenvalue 1 with multiplicity at least $L_{1}-L_{2}$. Moreover, all other positive eigenvalues of this sum must come in pairs $(2-\lambda, \lambda)$, where $1 \leq \lambda \leq \alpha<2$. Thus, by Lemma 3.2(v), $L_{1}+L_{2} \leq N$. Let $S=\alpha \mathbf{I}-P_{1}-P_{2}$. By Theorem 3.1, $S$ must satisfy (3-1). Note that the trace of $S$ remains constant regardless of choices of $P_{1}$ and $P_{2}$ :

$$
\operatorname{trace}(S)=\alpha N-L_{1}-L_{2} .
$$

Thus, the rank of $S$ must be minimized to guarantee that it can be written as a sum of projections. The minimal rank of $S$ occurs if $P_{1}+P_{2}$ has eigenvalue $\alpha$ with multiplicity $L_{2}$, and thus eigenvalue $2-\alpha$ with the same multiplicity. Then, the rank of the corresponding $S$ is $N-L_{2}$. Thus, we have

$$
\alpha N-L_{1}-L_{2} \geq N-L_{2} .
$$

This leads again to (3-3). Therefore, Fillmore's theorem does not introduce new constraints in this case. In other words, (3-3) and (3-4) are both necessary and sufficient conditions for the existence of an element of $\operatorname{TFF}(\alpha, N)$ starting with $\left(L_{1}, L_{2}\right)$.

Suppose next that $1<\alpha<3 / 2$. Repeating the above arguments, by Lemma 3.2, $P_{1}+P_{2}$ must have all of its $L_{1}+L_{2}$ nonzero eigenvalues (counted with multiplicities) in the interval $[2-\alpha, \alpha]$. Thus, if $L_{1}+L_{2}+L_{3}>N$, then at least one eigenvalue of $P_{1}+P_{2}+P_{3}$ would be at least $(2-\alpha)+1>3 / 2>\alpha$, which is impossible. Thus, (3-5) is necessary. 
To prove the converse, assume that $L_{1}+L_{2}+L_{3} \leq N$. Using honeycomb models as in the proof of Lemma 3.2, one can show that there exist projections $P_{i}$ such that their sum $P_{1}+P_{2}+P_{3}$ has the eigenvalue $\alpha$ with multiplicity $L_{2}+L_{3}$, and no eigenvalues bigger than $\alpha$. This is shown in a two step process. First, we construct $P_{1}$ and $P_{2}$ such that their sum has eigenvalues: $\alpha$ and $2-\alpha$ both with multiplicities $L_{2}$ and 1 with multiplicity $L_{1}-L_{2}$. Then, using a honeycomb model we can add on another projection $P_{3}$, such that $P_{1}+P_{2}+P_{3}$ has eigenvalue $\alpha$ with multiplicity $L_{2}+L_{3}$. See Figure 3 for an illustration of this honeycomb construction. We now have an operator $S=\alpha \mathbf{I}-\left(P_{1}+P_{2}+P_{3}\right)$ with the rank $N-L_{2}-L_{3}$. The trace of $S$ remains constant regardless of the choice of such projections:

$$
\operatorname{trace}(S)=\alpha N-L_{1}-L_{2}-L_{3} .
$$

Since $L_{1} \leq(\alpha-1) N$, Fillmore's Theorem 3.1 can be applied to represent $S$ as a sum of projections. This proves that (3-3)-(3-5) are both necessary and sufficient conditions for the first 3 ranks of a TFF sequence in the case $1<\alpha<3 / 2$.

In the special case $\alpha=3 / 2$, it is easy to see that $(N / 2, N / 2, N / 2)$ is the unique maximal element in $\operatorname{TFF}(\alpha, N)$; see Theorem 3.4. Unfortunately, the case $3 / 2<\alpha<2$ does not seem to be easily approachable with the techniques of this section. Instead, in Section 5 we shall give another combinatorial proof of Theorem 3.3 which works in the entire range $1<\alpha<2$.

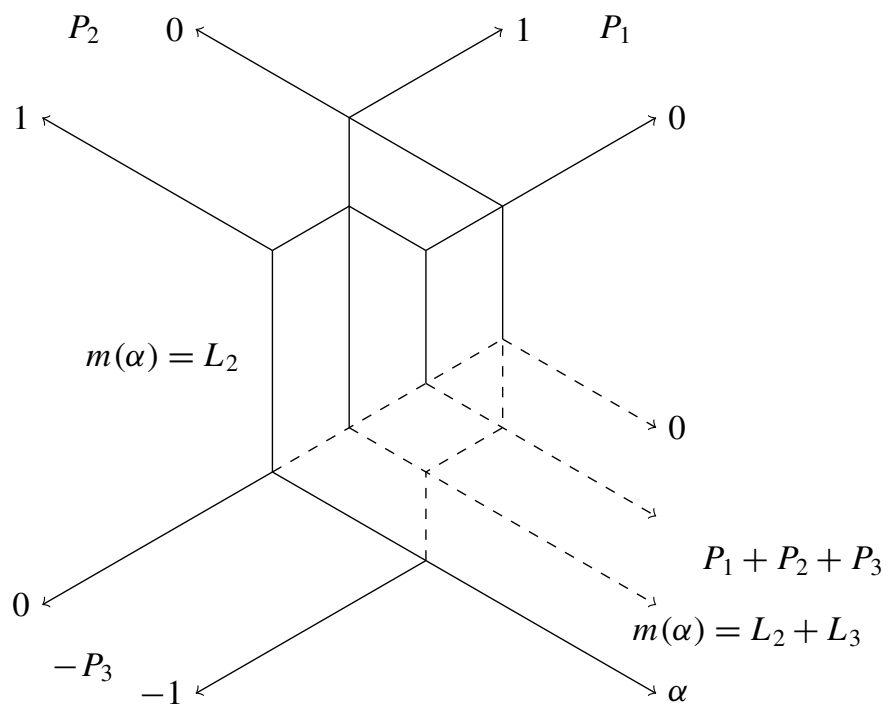

Figure 3. Honeycomb of $P_{1}+P_{2}+P_{3}$ with maximum eigenvalue $\alpha$ of multiplicity $L_{2}+L_{3}$. 
We end this section by an explicit characterization of TFF sequences for some special values $\alpha$.

Theorem 3.4. The set $\operatorname{TFF}(\alpha, N)$ has exactly one maximal element $\mathbf{L}$ with respect to majorization relation $\preccurlyeq$ in the following four cases indexed by $n \in \mathbb{N}$ :

$$
\begin{gathered}
\alpha=n, \quad \mathbf{L}=(\underbrace{N, N, \ldots, N}_{n}), \\
\alpha=1+\frac{1}{n}, \quad n \mid N, \quad \mathbf{L}=(\underbrace{\frac{N}{n}, \frac{N}{n}, \ldots, \frac{N}{n}}_{n+1}), \\
\alpha=n+\frac{1}{2}, \quad 2 \mid N, \quad \mathbf{L}=(\underbrace{N, \ldots, N}_{n-1}, \frac{N}{2}, \frac{N}{2}, \frac{N}{2}), \\
\alpha=1+\frac{2}{2 n-1}, \quad(2 n-1) \mid N, \quad \mathbf{L}=(\underbrace{\left.\frac{2 N}{2 n-1}, \ldots, \frac{2 N}{2 n-1}, \frac{N}{2 n-1}, \frac{N}{2 n-1}, \frac{N}{2 n-1}\right) .}_{n-1}
\end{gathered}
$$

Proof. The case (3-6) is the easiest and it follows immediately from Theorem 2.3. The case (3-7) is obtained by the duality argument. Indeed, note that if $\alpha=1+1 / n$, then $n$ must divide $N$. Then, by Corollary $2.7, \operatorname{TFF}(\alpha, N)=\operatorname{TFF}(\tilde{\alpha}, \tilde{N})$, where $\tilde{\alpha}=\alpha /(\alpha-1)=n+1$ and $\tilde{N}=(\alpha-1) N=N / n$.

In particular, we have that $\operatorname{TFF}(3 / 2, N)=\operatorname{TFF}(3, N / 2)$ has a unique maximal element $(N / 2, N / 2, N / 2)$. By appending $(n-1) N$ 's in the front of this sequence we obtain a maximal element of $\operatorname{TFF}(n+1 / 2, N)$. It remains to show that this is the only maximal element.

Suppose that we have another element $\left(L_{1}, \ldots, L_{K}\right) \in \operatorname{TFF}(n+1 / 2, N)$. Let $P_{i}$ 's be the corresponding projections. Given two hermitian matrices $S$ and $T$ we write $S \leq T$ if $\langle S x, x\rangle \leq\langle T x, x\rangle$ for all $x \in \mathbb{R}^{N}$. Since $\sum_{i=1}^{n} P_{i} \leq n \mathbf{I}, S=\sum_{i=n+1}^{K} P_{i}$ must have full rank $N$. By Fillmore's Theorem 3.1, this implies that

$$
\operatorname{trace}(S)=\sum_{i=n+1}^{K} L_{i} \geq N .
$$

Thus, $L_{1}+\cdots+L_{n} \leq(n-1 / 2) N$.

Suppose on the contrary that $L_{1}+\cdots+L_{n+1}>n N$. For each $i$, let $W_{i}$ be the corresponding subspace with $\operatorname{dim} W_{i}=L_{i}$. By basic linear algebra, the intersection satisfies

$$
\operatorname{dim}\left(\bigcap_{i=1}^{n+1} W_{i}\right) \geq L_{1}+\cdots+L_{n+1}-n N>0 .
$$


This implies that $P_{1}+\cdots+P_{n+1}$ has eigenvalue $n+1$ exceeding $\alpha=n+1 / 2$, which is a contradiction. Thus, we have necessarily $L_{1}+\cdots+L_{n+1} \leq n N$. Clearly,

$$
L_{1}+\cdots+L_{n+2} \leq L_{1}+\cdots+L_{K}=(n+1 / 2) N .
$$

Consequently, $\left(L_{1}, \ldots, L_{K}\right) \preccurlyeq \mathbf{L}$, proving (3-8).

Finally, case (3-9) is shown by the duality argument. If $\alpha=1+2 /(2 n-1)$, then $2 n-1$ must divide $N$. Then, by Corollary $2.7, \operatorname{TFF}(\alpha, N)=\operatorname{TFF}(\tilde{\alpha}, \tilde{N})$, where $\tilde{\alpha}=\alpha /(\alpha-1)=n+1 / 2$ and $\widetilde{N}=(\alpha-1) N=2 N /(2 n-1)$.

Section 7 provides the list of all maximal elements in $\operatorname{TFF}(\alpha, N)$ for all $\alpha \leq 2$ and dimensions $N \leq 9$. It is easy to observe that all unique maximal elements in our tables are covered by Theorem 3.4. Hence, it is very tempting to conjecture that for general $\alpha$ and $N$, if $\operatorname{TFF}(\alpha, N)$ has only one maximal element, then $\alpha$ must necessarily come from one of the four cases of Theorem 3.4.

\section{A combinatorial characterization of tight fusion frames}

In this section we give a combinatorial characterization of tight fusion frames in the context of Schur functions. The main result of this section, Theorem 4.3, is a direct consequence of Horn's recursion for the hermitian eigenvalue problem (for a survey of this problem see [Fulton 2000]). For completeness, we state the main results of this body of work. For any partition

$$
\lambda=\left(\lambda_{1} \geq \lambda_{2} \geq \cdots \geq \lambda_{d}>0\right),
$$

let

$$
|\lambda|=\sum_{i=1}^{d} \lambda_{i}
$$

denote the size of $\lambda$ and let $d$ denote the length. We say $\lambda$ is a rectangular partition if

$$
\lambda=\left(a^{b}\right):=(\underbrace{a, \ldots, a}_{b})
$$

for some positive integers $a, b$. For any partition $\lambda$, let $s_{\lambda}$ denote the corresponding Schur polynomial. The polynomial $s_{\lambda}$ is a homogeneous polynomial of degree $|\lambda|$. It is well known that the Schur polynomials form a linear basis of the algebra of symmetric polynomials with integer coefficients. Hence for any collection of partitions $\lambda^{1}, \ldots, \lambda^{K}$ we can define the corresponding Littlewood-Richardson coefficients $c\left(\lambda^{1}, \ldots, \lambda^{K} ; \mu\right)$ as the product structure constants of

$$
\prod_{i=1}^{K} s_{\lambda^{i}}=\sum_{\mu} c\left(\lambda^{1}, \ldots, \lambda^{K} ; \mu\right) s_{\mu} .
$$


The Littlewood-Richardson coefficients defined above play an important role in the hermitian eigenvalue problem. To state these results, we first need some notation. There is a standard identification between sets of positive integers of size $r$ and partitions of length at most $r$. For any set $I=\left\{i_{1}<i_{2}<\cdots<i_{r}\right\}$, define the partition

$$
\lambda(I):=\left(i_{r}-r, i_{r-1}-r+1, \ldots, i_{1}-1\right) .
$$

Let $\left(\beta^{1}, \ldots, \beta^{K+1}\right) \in\left(\mathbb{R}^{N}\right)^{K+1}$ denote a collection of sequences where each $\beta^{i}:=\left(\beta_{1}^{i} \geq \cdots \geq \beta_{N}^{i}\right)$. The goal of the hermitian eigenvalue problem is to determine for which sequences $\left(\beta^{1}, \ldots, \beta^{K+1}\right)$ do there exist $N \times N$ hermitian matrices $H_{1}, \ldots, H_{K+1}$ such that the eigenvalues of $H_{i}$ are given by the sequence $\beta^{i}$ and

$$
\sum_{i=1}^{K} H_{i}=H_{K+1} .
$$

The following theorem, due to Klyachko, gives a remarkable characterization in terms of a collection of inequalities parametrized by nonzero Littlewood-Richardson coefficients.

Theorem 4.1 [Klyachko 1998]. Let $\left(\beta^{1}, \ldots, \beta^{K+1}\right) \in\left(\mathbb{R}^{N}\right)^{K+1}$ be a collection of sequences of nonincreasing real numbers such that

$$
\sum_{i=1}^{K} \sum_{j=1}^{N} \beta_{j}^{i}=\sum_{j^{\prime}=1}^{N} \beta_{j^{\prime}}^{K+1} .
$$

Then the following are equivalent:

(1) There exist $N \times N$ hermitian matrices $H_{1}, \ldots, H_{K+1}$ with spectra $\left(\beta^{1}, \ldots, \beta^{K+1}\right)$ such that

$$
\sum_{i=1}^{K} H_{i}=H_{K+1} .
$$

(2) For every $r<N$, the sequence $\left(\beta^{1}, \ldots, \beta^{K+1}\right)$ satisfies the inequality

$$
\sum_{i=1}^{K} \sum_{j \in I^{j}} \beta_{j}^{i} \geq \sum_{j^{\prime} \in I^{K+1}} \beta_{j^{\prime}}^{K+1}
$$

for every collection of subsets $I^{1}, \ldots, I^{K+1}$ of size $r$ of integers $\{1,2, \ldots, N\}$ where the Littlewood-Richardson coefficient

$$
c\left(\lambda\left(I^{1}\right), \ldots, \lambda\left(I^{K}\right) ; \lambda\left(I^{K+1}\right)\right) \neq 0 .
$$

The inequalities given in (4-1) are called Horn's inequalities and were initially defined in a very different way by Horn [1962]. While Horn's list of inequalities 
are, a priori, different than Klyachko's list (4-1), they were shown to be equivalent as a consequence of the saturation theorem of Knutson and Tao [1999]. What is amazing about this equivalence is that Horn's initial definition of the inequalities (4-1) uses a recursion unrelated to Littlewood-Richardson coefficients. Horn's recursion, in light of Theorem 4.1, can be stated as follows:

Theorem 4.2. Let $I^{1}, \ldots, I^{K+1}$ be subsets of size $r$ of integers $\{1,2, \ldots, N\}$ such that

$$
\sum_{i=1}^{K} \sum_{j=1}^{r} \lambda\left(I^{i}\right)_{j}=\sum_{j^{\prime}=1}^{r} \lambda\left(I^{K+1}\right)_{j^{\prime}} .
$$

The following are equivalent:

(1) The Littlewood-Richardson coefficient

$$
c\left(\lambda\left(I^{1}\right), \ldots, \lambda\left(I^{K}\right) ; \lambda\left(I^{K+1}\right)\right) \neq 0 .
$$

(2) There exist $r \times r$ hermitian matrices $H_{1}, \ldots, H_{K+1}$ that have spectra

$$
\left(\lambda\left(I^{1}\right), \ldots, \lambda\left(I^{K+1}\right)\right)
$$

such that

$$
\sum_{i=1}^{K} H_{i}=H_{K+1} .
$$

The recursion says that a collection of subsets $I^{1}, \ldots, I^{K+1}$ corresponds to a Horn inequality if and only if the corresponding collection of partitions are eigenvalues of some $r \times r$ hermitian matrices which satisfy (4-3). Hence Horn's inequalities can be defined recursively by induction on $N$. We also remark that (4-2) is a necessary condition for the corresponding Littlewood-Richardson coefficient to be nonzero since it is equivalent to the grading condition when multiplying Schur functions in the graded algebra of symmetric functions.

We now apply Theorem 4.2 to the case of tight fusion frames. Suppose that $\left(L_{1} \geq L_{2} \geq \cdots \geq L_{K}\right) \in \operatorname{TFF}(\alpha, N)$ and that $M:=\sum_{i=1}^{K} L_{i}$. Then there exist orthogonal projections $P_{1}, \ldots, P_{K}$ such that

$$
\sum_{i=1}^{K} N P_{i}=M \mathbf{I} .
$$

Since $P_{i}$ is an orthogonal projection, the spectrum of the hermitian matrix $N P_{i}$ is given by

$$
(\underbrace{N, \ldots, N}_{L_{i}}, \underbrace{0, \ldots, 0}_{N-L_{i}}) .
$$


Let $\left(N^{L_{i}}\right)$ denote the corresponding rectangular partition to the spectra above. The following is a direct corollary of Theorem 4.2.

Theorem 4.3. Fix an integer $N$ and let $\left(L_{1} \geq L_{2} \cdots \geq L_{K}\right)$ be a sequence of nonnegative integers such that $L_{1} \leq N$. Let $M:=\sum_{i=1}^{K} L_{i}$ and $\alpha=M / N$. The following are equivalent:

(1) The sequence $\left(L_{1} \geq L_{2} \geq \cdots \geq L_{K}\right) \in \operatorname{TFF}(\alpha, N)$.

(2) The Littlewood-Richardson coefficient

$$
c\left(\left(N^{L_{1}}\right), \ldots,\left(N^{L_{K}}\right) ;\left(M^{N}\right)\right) \neq 0 .
$$

Proof. Assume part (1). Then there exist orthogonal projections $P_{1}, \ldots, P_{K}$ with ranks $\left(L_{1}, \ldots, L_{K}\right)$ such that

$$
\sum_{i=1}^{K} P_{i}=\alpha \mathbf{I} .
$$

Multiplying both sides of (4-5) by $N$ gives (4-4). Applying Theorem 4.2 gives part (2).

Conversely, if we assume part (2) then by Theorem 4.2, there exists a collection of $N \times N$ matrices which satisfy (4-4) and have spectra $\left(N^{L_{1}}\right), \ldots,\left(N^{L_{K}}\right)$. Scaling by $1 / N$ yields the desired tight fusion frame.

The condition that $c\left(\left(N^{L_{1}}\right), \ldots,\left(N^{L_{K}}\right) ;\left(M^{N}\right)\right) \neq 0$ can be made computationally explicit by the following existence condition. With the notation of Theorem 4.3 we consider the following properties for an $N \times M$ matrix $A=A[i, j]$.

(i) integral nonnegativity: $A[i, j] \in \mathbb{Z}_{\geq 0}$

(ii) row sum: $\sum_{j=1}^{M} A[i, j]=M$ for all $i$

(iii) column sum: $\sum_{i=1}^{N} A[i, j]=N$ for all $j$

(iv) row sum dominance: $\sum_{j=1}^{l}(A[i, j]-A[i+1, j]) \geq A[i+1, l+1]$ for all $i, l$

(v) column sum dominance: $\sum_{i=1}^{l}(A[i, j]-A[i, j+1]) \geq A[l+1, j+1]$ for all $j, l$

Note that we can take $l$ to be zero in conditions (iv) and (v) since in this case the sums are by definition equal to zero. Observe that properties (iv) and (v) require dominance with one additional summand in the later row or column. Also note that (ii) and (iii) are the only properties dependent on the size of the matrix $A$. Let $A$ be 
an $N \times M$ matrix and consider the sequence $\left(L_{1}, \ldots L_{K}\right)$. We can partition $A$ into a sequence of column block matrices

$$
A=\left[A_{1}\left|A_{2}\right| \cdots \mid A_{K}\right]
$$

where each $A_{i}$ is the corresponding $N \times L_{i}$ submatrix of $A$. We now have the following addition to Theorem 4.3.

Corollary 4.4. Conditions (1) and (2) in Theorem 4.3 are equivalent to:

(3) There exists an $N \times M$ matrix A which satisfies properties (i)-(iv) and whose column block submatrices $A_{1}, \ldots, A_{K}$ each satisfy property (v).

Moreover, the coefficient $c\left(\left(N^{L_{1}}\right), \ldots,\left(N^{L_{K}}\right) ;\left(M^{N}\right)\right)$ equals the number of $N \times M$ matrices A which satisfy (3).

Proof. We refer to [Fulton 1997] for definitions and details of Littlewood-Richardson skew tableaux. Consider the Littlewood-Richardson coefficients $c_{\lambda, \mu}^{v}$ corresponding to the product of two Schur functions

$$
s_{\lambda} s_{\mu}=\sum_{\nu} c_{\lambda, \mu}^{\nu} s_{\nu}
$$

It is well known that the number $c_{\lambda, \mu}^{v}$ is precisely equal to the number of Littlewood-Richardson skew tableaux $\nu / \lambda$ of content $\mu$. Now suppose there exists an $N \times M$ matrix $A$ which satisfies the conditions of Corollary 4.4 with respect to a sequence $\mathbf{L}=\left(L_{1}, \ldots, L_{K}\right)$. For any $k \leq K$ let

$$
A(k):=\left[A_{1}|\cdots| A_{k}\right]
$$

denote the submatrix of $A$ consisting of the matrices $A_{1}, \ldots, A_{k}$. By properties (i) and (iv), the row sums of $A(k)$ yield a partition

$$
\mu^{k}:=\left(\sum_{j} A(k)[i, j]\right)_{i=1}^{N}
$$

given in the standard weakly decreasing form. It is easy to see that $\mu^{k} / \mu^{k-1}$ is a well defined skew partition. Consider the Young diagram corresponding to $\mu^{k} / \mu^{k-1}$. We can fill the boxes of the $j$-th row of this diagram with $A_{k}[j, 1] 1 \mathrm{~s}$, $A_{k}[j, 2] 2 \mathrm{~s}, A_{k}[j, 3] 3$ 's and so forth in weakly increasing order. Property (iv) implies that the shape of $\mu^{k} / \mu^{k-1}$ is a valid skew partition and that the entries in each column are strictly decreasing. Property (v) implies that the row reading word is reverse lattice. Together this implies the resulting skew tableau is a LittlewoodRichardson skew tableau. Property (iii) implies that content of the tableau is that 
of the rectangular partition $\left(N^{L_{k}}\right)$. Hence the existence of the matrix $A(k)$ implies that the Littlewood-Richardson coefficient

$$
c_{\mu^{k-1},\left(N^{\left.L_{k}\right)}\right.}^{\mu^{k}} \neq 0
$$

Properties (ii) and (iii) imply that $\mu^{K}=\left(M^{N}\right)$. By induction on $k$, multiplying the Schur functions $s_{\left(N^{L_{1}}\right)}, \ldots s_{\left(N^{\left.L_{K}\right)}\right.}$ gives

$$
c\left(\left(N^{L_{1}}\right), \ldots,\left(N^{L_{K}}\right) ;\left(M^{N}\right)\right) \neq 0 .
$$

Conversely, if the second part of Theorem 4.3 holds, then there exists a sequence of partitions $\mu^{1}, \mu^{2}, \ldots, \mu^{K}$ such that (4-7) holds with $\mu^{1}=\left(N^{L_{1}}\right)$ and $\mu^{K}=\left(M^{N}\right)$. In particular, there exists a Littlewood-Richardson skew tableau of shape $\mu^{k} / \mu^{k-1}$ with content $\left(N^{L_{k}}\right)$. We can construct a matrix $A_{k}$ which satisfies property $(v)$ using the entries of this Littlewood-Richardson skew tableau by reversing the argument above. Moreover, taking $A=\left[A_{1}|\cdots| A_{K}\right]$, we have that $A$ satisfies all the conditions part (3) of Corollary 4.4.

Finally, the Littlewood-Richardson rule states that

$$
c_{\mu^{k-1},\left(N^{L_{k}}\right)}^{\mu^{k}}
$$

is precisely the number of Littlewood-Richardson skew tableaux of shape $\mu^{k} / \mu^{k-1}$ with content $\left(N^{L_{k}}\right)$. Hence, the second part of Corollary 4.4 follows from the bijection given by (4-6). This completes the proof.

Example 4.5. We consider two examples where tight fusion frames exist for $N=5$ and $M=8$.

First, consider the sequence $\mathbf{L}=(2,2,2,2)$. The matrix

$$
A=\left(\begin{array}{llllllll}
5 & 0 & 3 & 0 & 0 & 0 & 0 & 0 \\
0 & 5 & 0 & 1 & 2 & 0 & 0 & 0 \\
0 & 0 & 2 & 2 & 2 & 2 & 0 & 0 \\
0 & 0 & 0 & 2 & 1 & 0 & 5 & 0 \\
0 & 0 & 0 & 0 & 0 & 3 & 0 & 5
\end{array}\right)
$$

satisfies the conditions in Corollary 4.4. We write out the corresponding Young tableaux to the partitions $\mu^{1}, \mu^{2}, \mu^{3}$ and $\mu^{4}$ with content given by the submatrices 
$A(1), A(2), A(3), A(4)$ :

\begin{tabular}{|l|l|l|l|l|}
\hline 1 & 1 & 1 & 1 & 1 \\
\hline 2 & 2 & 2 & 2 & 2 \\
\hline
\end{tabular}

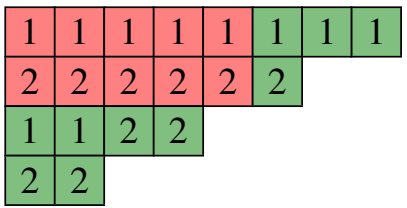

\begin{tabular}{|l|l|l|l|l|l|l|l|}
\hline 1 & 1 & 1 & 1 & 1 & 1 & 1 & 1 \\
\hline 2 & 2 & 2 & 2 & 2 & 2 & 1 & 1 \\
\hline 1 & 1 & 2 & 2 & 1 & 1 & 2 & 2 \\
\hline 2 & 2 & 1 & \multicolumn{7}{|c|}{} \\
\cline { 1 - 2 } 2 & 2 & 2 & & \multicolumn{1}{|c}{} \\
\cline { 1 - 2 } & & & & & \\
\end{tabular}

\begin{tabular}{|l|l|l|l|l|l|l|l|}
\hline 1 & 1 & 1 & 1 & 1 & 1 & 1 & 1 \\
\hline 2 & 2 & 2 & 2 & 2 & 2 & 1 & 1 \\
\hline 1 & 1 & 2 & 2 & 1 & 1 & 2 & 2 \\
\hline 2 & 2 & 1 & 1 & 1 & 1 & 1 & 1 \\
\hline 2 & 2 & 2 & 2 & 2 & 2 & 2 & 2 \\
\hline
\end{tabular}

Note that the all the data can be encoded in the final partition $\mu^{4}$ as a union of skew Littlewood-Richardson tableaux.

For the second example, we consider $\mathbf{L}=(3,2,1,1,1)$, the matrix $A$ and the corresponding union of Littlewood-Richardson tableaux

$$
A=\left(\begin{array}{lll|ll|l|l|l}
5 & 0 & 0 & 3 & 0 & 0 & 0 & 0 \\
0 & 5 & 0 & 0 & 3 & 0 & 0 & 0 \\
0 & 0 & 5 & 0 & 0 & 3 & 0 & 0 \\
0 & 0 & 0 & 2 & 0 & 2 & 4 & 0 \\
0 & 0 & 0 & 0 & 2 & 0 & 1 & 5
\end{array}\right)
$$

\begin{tabular}{|l|l|l|l|l|l|l|l|}
\hline 1 & 1 & 1 & 1 & 1 & 1 & 1 & 1 \\
\hline 2 & 2 & 2 & 2 & 2 & 2 & 2 & 2 \\
\hline 3 & 3 & 3 & 3 & 3 & 1 & 1 & 1 \\
\hline 1 & 1 & 1 & 1 & 1 & 1 & 1 & 1 \\
\hline 2 & 2 & 1 & 1 & 1 & 1 & 1 & 1 \\
\hline
\end{tabular}

We end this section with an important observation concerning the partial sum of orthogonal projections $P_{1}+\cdots+P_{k}$. Let $A$ be a matrix as in Corollary 4.4 for some $\mathbf{L} \in \operatorname{TFF}(\alpha, N)$ and consider the sequence of partitions $\mu^{1}, \ldots, \mu^{K}$ corresponding to $A$. For each $k \leq K$, we have that the coefficient

$$
c\left(\left(N^{L_{1}}\right), \ldots,\left(N^{L_{k}}\right) ; \mu^{k}\right) \neq 0 .
$$

This fact together with Theorem 4.2 yields the following corollary

Corollary 4.6. Let $A$ be a matrix as in Corollary 4.4 for some $\mathbf{L} \in \operatorname{TFF}(\alpha, N)$ and consider the sequence of partitions $\mu^{1}, \ldots, \mu^{K}$ corresponding to A. Then there exist orthogonal projections $P_{1}, \ldots, P_{K}$ such that (4-4) holds and each partial sum $\sum_{i=1}^{k} P_{i}$ has spectrum $\left(\mu_{1}^{k} / N, \ldots, \mu_{N}^{k} / N\right)$.

We remark that Corollary 4.6 is useful for the construction of explicit tight fusion frames. We illustrate this process later in Example 7.2. 


\section{Combinatorial majorization and hook type sequences}

In this section we give alternate proofs of Theorem 2.3 on majorization as well as Theorem 3.3 on estimates using the combinatorics of Schur functions and Theorem 4.3. We begin with some fundamental definitions and lemmas on Schur functions. Let $\lambda \subseteq\left(M^{N}\right)$. We define the dual partition of $\lambda$ in $\left(M^{N}\right)$ to be

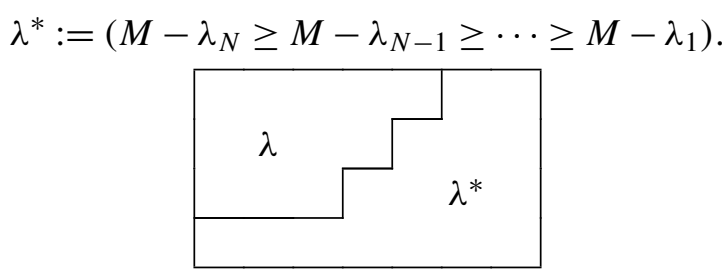

Lemma 5.1. Let $\lambda \subseteq\left(M^{N}\right)$ and let $p(\lambda)$ denote the number of parts of $\lambda$ equal to $M$. Assume that for some positive integer $k$ we have

$$
|\lambda|=N(M-k) .
$$

Then

if and only if $k \geq N-p(\lambda)$.

$$
c(\lambda, \underbrace{(N), \ldots,(N)}_{k} ;\left(M^{N}\right)) \neq 0
$$

Proof. The lemma follows from two elementary facts about Schur functions. Consider the product

$$
\left(s_{(N)}\right)^{k}=\sum_{\mu} c((N), \ldots,(N) ; \mu) s_{\mu}
$$

By the Pieri rule, we have that $c((N), \ldots,(N) ; \mu) \neq 0$ if and only if $\mu$ has length less than or equal to $k$ and $|\mu|=N k$. Furthermore, if $\lambda, \mu \subseteq\left(M^{N}\right)$, then $c_{\lambda, \mu}^{\left(M^{N}\right)} \neq 0$ if and only if $\mu=\lambda^{*}$. It is easy to check that $\lambda^{*}$ appears as a summand in the product $\left(s_{(N)}\right)^{k}$ precisely when $k \geq N-p(\lambda)$.

The following theorem on the product of Schur functions corresponding to rectangular partitions is proved by Okada.

Theorem 5.2 [Okada 1998, Theorem 2.4]. Fix integers $a, b, N_{1}, N_{2}$ with $a \geq b$. The product of Schur functions

$$
s_{\left(N_{1}^{a}\right) s_{\left(N_{2}^{b}\right)}}=\sum_{\lambda} s_{\lambda},
$$

where the sum is over all partitions $\lambda$ with length $\leq a+b$ such that

- $\lambda_{b+1}=\lambda_{b+2}=\cdots=\lambda_{a}=N_{1}$,

- $\lambda_{b} \geq \max \left\{N_{1}, N_{2}\right\}$,

- $\lambda_{i}+\lambda_{a+b+1-i}=N_{1}+N_{2} \quad$ for all $i \in\{1, \ldots, b\}$. 
We now give an alternate proof of Theorem 2.3 using Theorem 5.2 in the case when $N_{1}=N_{2}$.

Lemma 5.3. Fix a positive integer $N$ and let $0<a<b$. Then the LittlewoodRichardson coefficients

$$
c_{\left(N^{b}\right),\left(N^{a}\right)}^{\lambda} \leq c_{\left(N^{b-1}\right),\left(N^{a+1}\right)}^{\lambda} .
$$

In particular, Theorem 2.3 on majorization of tight fusion frames follows.

Proof. It is easy to check the $\lambda$ that appear in the summation (5-1) for the pair $\left(\left(N^{b}\right),\left(N^{a}\right)\right)$ are contained in the $\lambda$ that appear in the summation (5-1) for the pair $\left(\left(N^{b-1}\right),\left(N^{a+1}\right)\right)$. This proves the inequality. The application to tight fusion frames follows from Theorem 4.3.

It is easy to see that by majorization, the following theorem is equivalent to Theorem 3.3 on estimates.

Theorem 5.4. Assume the conditions in Theorem 4.3. Further assume that $\alpha=$ $M / N<2$. If $\left(L_{1} \geq L_{2} \geq \cdots \geq L_{K}\right) \in \operatorname{TFF}(\alpha, N)$, then we have the following necessary conditions:

(1) $L_{1} \leq M-N$.

(2) $L_{1}+L_{2} \leq N$.

(3) If $\alpha>3 / 2$, then $L_{1}+L_{2}+L_{3} \leq 2(M-N)$.

(4) If $\alpha<3 / 2$, then $L_{1}+L_{2}+L_{3} \leq N$.

Conversely, suppose $L_{1}, L_{2}, L_{3}$ satisfy conditions (1)-(4) and $L_{4}=\cdots=L_{K}=1$. Then $\left(L_{1} \geq L_{2} \geq \cdots \geq L_{K}\right) \in \operatorname{TFF}(\alpha, N)$.

Proof. Recall that for any partition $\lambda \subseteq\left(M^{N}\right)$, we let $p(\lambda)$ denote the number of parts of $\lambda$ equal to $M$. First we prove part (1). By majorization, it suffices to assume that $L_{2}=1$. Part (1) now follows from Lemma 5.1 by setting $\lambda=\left(N^{L_{1}}\right)$ and observing that $p\left(\left(N^{L_{1}}\right)\right)=0$.

We now prove part (2). By majorization, it suffices to assume that $L_{3}=1$. Consider the product

$$
s_{\left(N^{\left.L_{1}\right)}\right.} S_{\left(N^{\left.L_{2}\right)}\right.}=\sum_{\lambda} s_{\lambda}
$$

By Theorem 5.2, we have that $\lambda_{1}+\lambda_{L_{1}+L_{2}}=2 N$ for every $\lambda$ in the sum (5-2). If $\lambda \subseteq\left(M^{N}\right)$, then $\lambda_{1} \leq M$. Note that such a partition $\lambda$ exists since $\left(L_{1} \geq L_{2} \geq\right.$ $\left.\cdots \geq L_{K}\right) \in \operatorname{TFF}(\alpha, N)$. Hence

$$
\lambda_{L_{1}+L_{2}}=2 N-\lambda_{1} \geq 2 N-M>0
$$

since $\alpha<2$. This implies that $L_{1}+L_{2} \leq N$ since $\left(M^{N}\right)$ has only $N$ parts. 
For part (3), we assume that $L_{4}=1$. First, if $L_{2}+L_{3} \leq L_{1}$, then by part (1), $L_{1}+L_{2}+L_{3} \leq 2(M-N)$. Next, we assume $L_{1} \leq L_{2}+L_{3}$. Consider the product

$$
s_{\left(N^{L_{1}}\right)} S_{\left(N^{\left.L_{2}\right)}\right.} S_{\left(N^{\left.L_{3}\right)}\right.}=\sum_{\lambda} c\left(\left(N^{L_{1}}\right),\left(N^{L_{2}}\right),\left(N^{L_{3}}\right) ; \lambda\right) s_{\lambda} .
$$

Since $\alpha>3 / 2$, for any $\lambda \subseteq\left(M^{N}\right)$ such that $c\left(\left(N^{L_{1}}\right),\left(N^{L_{2}}\right),\left(N^{L_{3}}\right) ; \lambda\right) \neq 0$, we have $p(\lambda) \leq L_{1}$. This can be seen by considering $L_{2}$ and $L_{3}$ as large as possible, hence $L_{1}=L_{2}=L_{3}$. One can show using the Littlewood-Richardson rule that since $3 N<2 M$, three layered bricks of width $N$ cannot span $M$ more than once; see diagram below.

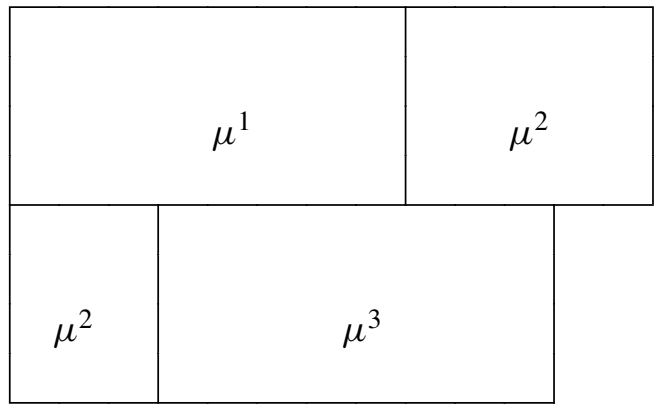

By Lemma 5.1,

$$
M-L_{1}-L_{2}-L_{3} \geq N-p(\lambda) \geq N-L_{1} .
$$

Hence $L_{2}+L_{3} \leq M-N$. This proves part (3).

For part (4), fix any $\lambda$ in the summand found in (5-2) such that $\lambda \subseteq\left(M^{N}\right)$. Since $\alpha<3 / 2$, we have

$$
\lambda_{L_{1}+L_{2}}=2 N-\lambda_{1} \geq 2 N-M>M-N .
$$

Hence the rectangular partition $\left((M-N+1)^{L_{1}+L_{2}}\right) \subseteq \lambda$. Comparing the two products

$$
s_{\lambda} s_{\left(N^{\left.L_{3}\right)}\right.}=\sum_{\mu^{\prime}} c_{\lambda,\left(N^{\left.L_{3}\right)}\right.}^{\mu^{\prime}} s_{\mu^{\prime}}
$$

and

$$
S_{\left((M-N+1)^{\left.L_{1}+L_{2}\right)}\right.} S_{\left(N^{\left.L_{3}\right)}\right.}=\sum_{\mu} s_{\mu}
$$

we have that any partition $\mu^{\prime}$ from (5-4) such that $c_{\lambda,\left(N^{\left.L_{3}\right)}\right.}^{\mu^{\prime}} \neq 0$ contains some $\mu$ from (5-5). Therefore it is enough to consider the partitions $\mu$ from (5-5). By Theorem 5.2, we get

$$
\mu_{1}+\mu_{L_{1}+L_{2}+L_{3}}=M-N+1+N=M+1
$$


for every $\mu$ in the sum (5-5). Hence if $\mu \subseteq\left(M^{N}\right)$, then $\mu_{L_{1}+L_{2}+L_{3}}>0$ since $\mu_{1} \leq M$. Note that such a partition $\mu$ exists since $\left(L_{1} \geq L_{2} \geq \cdots \geq L_{K}\right) \in \operatorname{TFF}(\alpha, N)$. Thus $L_{1}+L_{2}+L_{3} \leq N$. This proves part (4).

To prove sufficiency, we construct $\lambda$ in the sum (5-3) such that $\lambda \subseteq\left(M^{N}\right)$ and $c\left(\left(N^{L_{1}}\right),\left(N^{L_{2}}\right),\left(N^{L_{3}}\right) ; \lambda\right) \neq 0$. One can show using the Littlewood-Richardson rule that parts (1)-(4) imply that such a $\lambda$ exists. Furthermore, we can construct $\lambda$ such that $p(\lambda)$ satisfies the following:

- If $L_{1} \geq L_{2}+L_{3}$, then $p(\lambda)=L_{2}+L_{3}$.

- If $L_{1}<L_{2}+L_{3}$ and $\alpha<3 / 2$, then $p(\lambda)=L_{2}+L_{3}$.

- If $L_{1}<L_{2}+L_{3}$ and $\alpha>3 / 2$, then $p(\lambda)=\left\lfloor\frac{1}{2}\left(L_{1}+L_{2}+L_{3}\right)\right\rfloor$.

See Figures 4 and 5 for a diagram of $\lambda$ in the cases above. If $p(\lambda)=L_{2}+L_{3}$, then Lemma 5.1 implies we need to check that $L_{1} \leq M-N$, which is a necessary condition. The last case is the most delicate. We construct $\lambda$ with a partition $\mu_{2}$ of the shape as in Figure 5 with a notch of height $x$. If $L_{1}+L_{2}+L_{3}$ is even, then we choose $x$ such that $p(\lambda)=x+L_{3}=L_{1}+L_{2}-x$, and hence $p(\lambda)=\frac{1}{2}\left(L_{1}+L_{2}+L_{3}\right)$. If $L_{1}+L_{2}+L_{3}$ is odd, then $p(\lambda)=\left\lfloor\frac{1}{2}\left(L_{1}+L_{2}+L_{3}\right)\right\rfloor$. Either way, Lemma 5.1 implies we need to check that $L_{1}+L_{2}+L_{3} \leq 2(M-N)$, which again is a necessary condition. This completes the proof of the theorem.

Remark 5.5. Parts (2) and (4) of Theorem 5.4 can be generalized to the following. Claim: Let $2 \leq k \leq K$. If $\alpha<k /(k-1)$, then $L_{1}+\cdots+L_{k} \leq N$. The proof follows the same argument as the proof of Theorem 5.4 part (4).
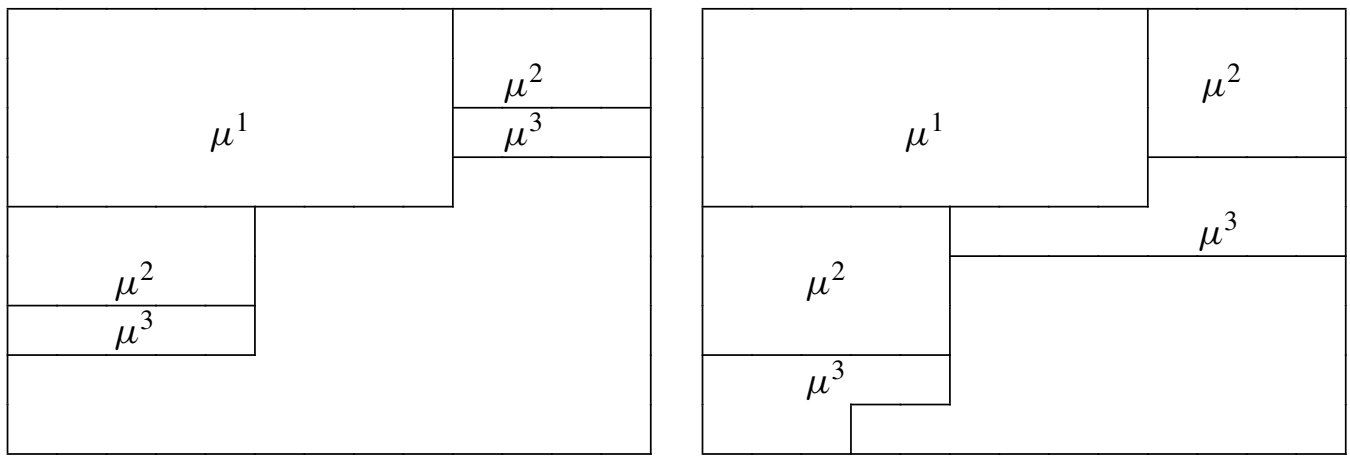

Figure 4. Construction of $\lambda$ for $\alpha<3 / 2$ as a union of LittlewoodRichardson skew tableaux $\mu^{1}, \mu^{2}, \mu^{3}$ when $L_{2}+L_{3} \leq L_{1}$ and $L_{1} \leq L_{2}+L_{3}$, respectively. This construction is possible since $L_{1}+L_{2}+L_{3} \leq N$ and $2 M<3 N$. 

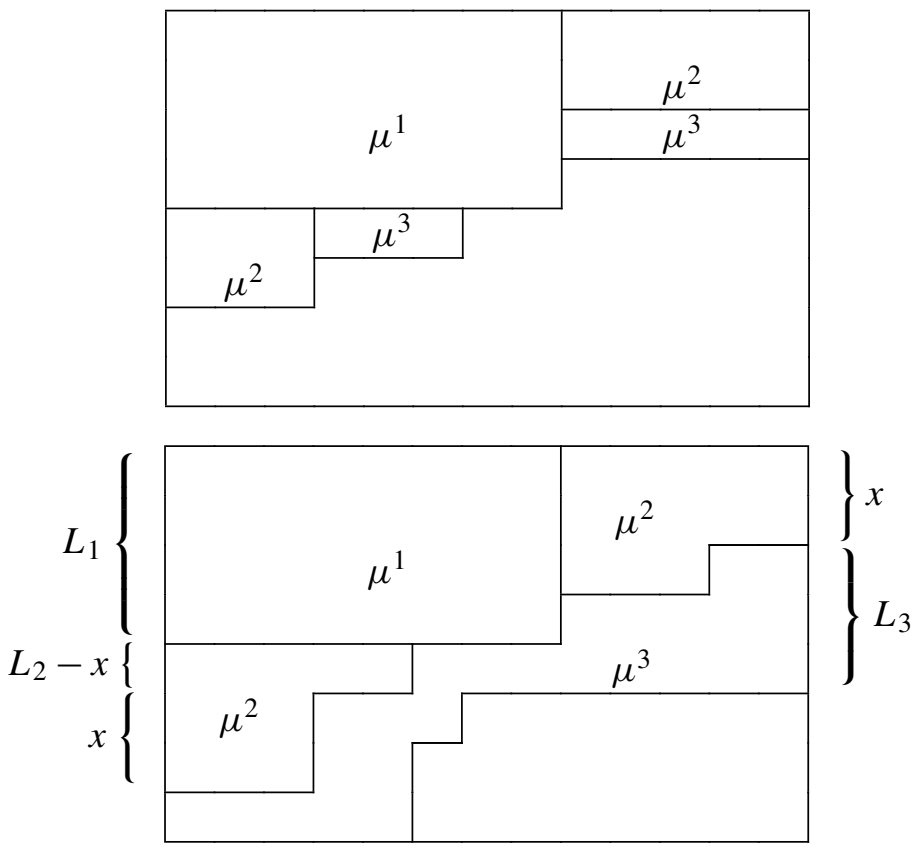

Figure 5. Construction of $\lambda$ for $\alpha>3 / 2$ as a union of LittlewoodRichardson skew tableaux $\mu^{1}, \mu^{2}, \mu^{3}$ when $L_{2}+L_{3} \leq L_{1}$ and $L_{1} \leq L_{2}+L_{3}$, resp.

\section{Combinatorial spatial and Naimark duality}

Theorems 2.5 and 2.6 establish spatial and Naimark dualities for tight fusion frames. By Theorem 4.3, we have the analogous results for Littlewood-Richardson coefficients.

Corollary 6.1. Assume we have a sequence of integers $\left(L_{1} \geq \cdots \geq L_{K}\right)$ as in Theorem 4.3. Then

$$
\begin{aligned}
c\left(\left(N^{L_{1}}\right), \ldots,\left(N^{L_{K}}\right) ;\left(M^{N}\right)\right) \neq 0 & \\
& \Longleftrightarrow c\left(\left(N^{N-L_{1}}\right), \ldots,\left(N^{N-L_{K}}\right) ;\left((K N-M)^{N}\right)\right) \neq 0
\end{aligned}
$$

and

$$
\begin{aligned}
c\left(\left(N^{L_{1}}\right), \ldots,\left(N^{L_{K}}\right) ;\left(M^{N}\right)\right) \neq 0 & \\
& \Longleftrightarrow c\left(\left((M-N)^{L_{1}}\right), \ldots,\left((M-N)^{L_{K}}\right) ;\left(M^{(M-N)}\right)\right) \neq 0 .
\end{aligned}
$$

In this section we prove a much stronger version of the corollary above. In particular, we prove that these Littlewood-Richardson coefficients are equal. We will frequently reference properties (i)-(v) for matrices defined in the paragraph 
preceding Corollary 4.4 using lower case roman numerals. We first consider spatial duality.

Theorem 6.2. The Littlewood-Richardson coefficients

(6-1) $\quad c\left(\left(N^{L_{1}}\right), \ldots,\left(N^{L_{K}}\right) ;\left(M^{N}\right)\right)=$

$$
c\left(\left(N^{N-L_{1}}\right), \ldots,\left(N^{N-L_{K}}\right) ;\left((K N-M)^{N}\right)\right) .
$$

The coefficient $c\left(\left(N^{L_{1}}\right), \ldots,\left(N^{L_{K}}\right) ;\left(M^{N}\right)\right)$ is precisely the number of $N \times M$ matrices $A$ which satisfy the conditions given in the Corollary 4.4. We will call such a collection of matrices the set of configuration matrices corresponding to $\left(L_{1}, \ldots, L_{K} ; N\right)$. We prove Theorem 6.2 by providing a bijection between the configuration matrices corresponding to the coefficients in (6-1).

Suppose that $c\left(\left(N^{L_{1}}\right), \ldots,\left(N^{L_{K}}\right) ;\left(M^{N}\right)\right) \neq 0$ and fix a configuration matrix $A=\left[A_{1}\left|A_{2}\right| \cdots \mid A_{K}\right]$. For each $A_{i}$, we construct an $N \times\left(N-L_{i}\right)$ matrix $B_{i}$ as follows. Decompose

$$
A_{i}=\sum_{j=1}^{N} C_{j}
$$

as a sum of binary matrices which for all integers $y, j$, satisfy

(1) $\sum_{x=1}^{N} C_{j}[x, y]=1$.

(2) $\sum_{x=1}^{N^{\prime}}\left(C_{j}[x, y]-C_{j}[x, y+1]\right) \geq 0$ for all $N^{\prime}<N$.

(3) $\sum_{x=1}^{N^{\prime}}\left(C_{j}[x, y]-C_{j+1}[x, y]\right) \geq 0 \quad$ for all $N^{\prime}<N$.

Consider $A_{2}$ from Example 4.5. We have

$$
\left(\begin{array}{ll}
3 & 0 \\
0 & 1 \\
2 & 2 \\
0 & 2 \\
0 & 0
\end{array}\right)=\left(\begin{array}{ll}
1 & 0 \\
0 & 1 \\
0 & 0 \\
0 & 0 \\
0 & 0
\end{array}\right)+\left(\begin{array}{ll}
1 & 0 \\
0 & 0 \\
0 & 1 \\
0 & 0 \\
0 & 0
\end{array}\right)+\left(\begin{array}{ll}
1 & 0 \\
0 & 0 \\
0 & 1 \\
0 & 0 \\
0 & 0
\end{array}\right)+\left(\begin{array}{ll}
0 & 0 \\
0 & 0 \\
1 & 0 \\
0 & 1 \\
0 & 0
\end{array}\right)+\left(\begin{array}{ll}
0 & 0 \\
0 & 0 \\
1 & 0 \\
0 & 1 \\
0 & 0
\end{array}\right) .
$$

It is easy to see that this decomposition of $A_{i}$ is unique since $A_{i}$ satisfies properties (i), (iii) and (v). For each $C_{j}$, define the $N \times\left(N-L_{i}\right)$ matrix $C_{j}^{\prime}$ to be the unique binary matrix which satisfies conditions (1), (2) and that $\left[C_{j} \mid C_{j}^{\prime}\right]$ is invertible. For 
example, if $N=5$ then

$$
C_{j}=\left(\begin{array}{ll}
1 & 0 \\
0 & 0 \\
0 & 1 \\
0 & 0 \\
0 & 0
\end{array}\right) \rightsquigarrow C_{j}^{\prime}=\left(\begin{array}{lll}
0 & 0 & 0 \\
1 & 0 & 0 \\
0 & 0 & 0 \\
0 & 1 & 0 \\
0 & 0 & 1
\end{array}\right) .
$$

Define

$$
B_{i}:=\sum_{i=1}^{N} C_{j}^{\prime}
$$

and consider the $N \times(K N-M)$ matrix

$$
B:=\left[B_{K}\left|B_{K-1}\right| \cdots \mid B_{1}\right] .
$$

Note that the binary decomposition of $B_{i}$ into $C_{j}^{\prime}$ also satisfies conditions (1)-(3) if we order the $C_{j}^{\prime}$ in reverse. Moreover, if we apply this algorithm to the matrix $B$, we will recover the matrix $A$. We now record some important observations on the submatrices $A_{i}$ and $B_{i}$. First, if $x<y$, then

$$
A_{i}[x, y]=B_{i}[x, y]=0 .
$$

Second,

$$
A_{i}[x, y]+B_{i}[x, x-y]=A_{i}[x+1, y+1]+B_{i}[x+1, x-y+1] .
$$

In the equations above we take $A_{i}[x, y]=0$ (respectively, $B_{i}[x, y]=0$ ) if $x, y$ lie outside the boundaries of $A_{i}$ (respectively, $B_{i}$ ). In the case when $x=y$, we get

$$
A_{i}[x, x]=A_{i}[x+1, x+1]+B_{i}[x+1,1] .
$$

Theorem 6.2 follows from the proceeding proposition.

Proposition 6.3. The $N \times(K N-M)$ matrix $B$ is a configuration matrix for the sequence $\left(N-L_{K}, \ldots, N-L_{1} ; K N-M\right)$.

Proof. The most challenging part of this proof is to show that the matrix $B$ satisfies property (iv). Hence the majority of this argument is dedicated to the proof this property. We first consider the other properties. Properties (i)-(iii) are immediate by construction of $B$. Property (v) follows from the fact that each $B_{i}$ is a sum of binary matrices which satisfy conditions (1)-(3). We now prove that $B$ satisfies property (iv) by contradiction. Suppose there exist integers $i, l$ such that

$$
\sum_{j=1}^{l}(B[i, j]-B[i+1, j])<B[i+1, l+1] .
$$


We define the integers $k, l^{\prime}$ as follows. Let $k$ denote largest integer for which the partial sum

$$
l^{\prime}:=\sum_{j=1}^{k}\left(N-L_{K-j+1}\right) \leq l
$$

Hence $l^{\prime}$ is the number of columns of the submatrix $\left[B_{K}|\cdots| B_{K-k+1}\right]$ of $B$.

Observe that each row sum of the matrix $\left[A_{j} \mid B_{j}\right]$ is equal to $N$. Combining this observation with (6-5) gives

$$
\begin{aligned}
\sum_{j=1}^{l}(B[i, j]- & B[i+1, j]) \\
& =\sum_{j=1}^{l^{\prime}}(B[i, j]-B[i+1, j])+\sum_{j=l^{\prime}+1}^{l}(B[i, j]-B[i+1, j]) \\
& =\sum_{j=M-\left(k N-l^{\prime}-1\right)}^{M}(A[i+1, j]-A[i, j])+\sum_{j=l^{\prime}+1}^{l}(B[i, j]-B[i+1, j]) \\
& <B[i+1, l+1] .
\end{aligned}
$$

Rewriting this inequality yields

$$
\begin{aligned}
& \sum_{j=M-k N+l^{\prime}+1}^{M}(A[i+1, j]-A[i, j]) \\
&<B[i+1, l+1]-\sum_{j=l^{\prime}+1}^{l}(B[i, j]-B[i+1, j]) \\
&=B\left[i+1, l^{\prime}+1\right]+\sum_{j=l^{\prime}+1}^{l}(B[i+1, j+1]-B[i, j]) .
\end{aligned}
$$

The matrix entries of $B$ appearing on the right hand side of the above equation are all contained in the submatrix $B_{K-k}$. Applying (6-3) and (6-4), we get $(6-6)$

$$
\sum_{j=M-k N+l^{\prime}+1}^{M}(A[i+1, j]-A[i, j])<\sum_{j=0}^{l-l^{\prime}}\left(A_{K-k}[i, i-j]-A_{K-k}[i+1, i-j+1]\right) .
$$


By (6-2), $A_{K-k}[x, y]=0$ if $y>x$. Hence we can extend the right hand side of (6-6) to

$$
\sum_{j=M-k N+l^{\prime}+1}^{M}(A[i+1, j]-A[i, j])
$$

$<A_{K-k}\left[i, i-l+l^{\prime}\right]+\sum_{j=0}^{L_{K-k}-(i+1)+\left(l-l^{\prime}\right)}\left(A_{K-k}\left[i, L_{K-k}-j\right]-A_{K-k}\left[i+1, L_{K-k}-j\right]\right)$.

Now the fact that $A$ satisfies properties (ii), contradicts the fact that it also satisfies property (iv). This completes the proof.

Example 6.4. Let $N=4$ and consider the sequence $\mathbf{L}=(2,2,2,1)$. Using Corollary 4.4, the matrix $A$ below implies that $\mathbf{L} \in \operatorname{TFF}(7 / 4,4)$.

$$
A=\left(\begin{array}{ll|ll|ll|l}
4 & 0 & 3 & 0 & 0 & 0 & 0 \\
0 & 4 & 0 & 1 & 2 & 0 & 0 \\
0 & 0 & 1 & 2 & 2 & 2 & 0 \\
0 & 0 & 0 & 1 & 0 & 2 & 4
\end{array}\right)
$$

We get

$$
B=\left(\begin{array}{lll|ll|ll|ll}
4 & 0 & 0 & 4 & 0 & 1 & 0 & 0 & 0 \\
0 & 4 & 0 & 0 & 2 & 2 & 1 & 0 & 0 \\
0 & 0 & 4 & 0 & 0 & 1 & 0 & 4 & 0 \\
0 & 0 & 0 & 0 & 2 & 0 & 3 & 0 & 4
\end{array}\right)
$$

and hence $(3,2,2,2) \in \operatorname{TFF}(9 / 4,4)$.

We now give the analogous theorem on combinatorial Naimark duality.

Theorem 6.5. The Littlewood-Richardson coefficients

$$
\begin{aligned}
& c\left(\left(N^{L_{1}}\right), \ldots,\left(N^{L_{K}}\right) ;\left(M^{N}\right)\right) \\
& \quad=c\left(\left((M-N)^{L_{1}}\right), \ldots,\left((M-N)^{L_{K}}\right) ;\left(M^{(M-N)}\right)\right) .
\end{aligned}
$$

As with Theorem 6.2, we define a bijection between configuration matrices corresponding to the Littlewood-Richardson coefficients in (6-7). Fix a configuration matrix $A$ corresponding to the sequence $\left(L_{1}, \ldots, L_{K} ; N\right)$ and consider the Littlewood-Richardson skew tableaux $\mu^{k} / \mu^{k-1}$ where $\mu^{k}$ is defined in (4-6). To each $\mu^{k} / \mu^{k-1}$ we define the $L_{k} \times M$ binary matrix $T_{k}$ by

$$
T_{k}[x, y]:= \begin{cases}1, & \text { if } x \text { appears in column } y \text { of } \mu^{k} / \mu^{k-1} \\ 0, & \text { otherwise. }\end{cases}
$$

The partition shape of $\mu^{k}$ can be recovered from the matrices $T_{1}, \ldots T_{K}$ as follows. Define the matrix $T(k)$ by "stacking" the matrices $T_{1}, \ldots, T_{k}$ (see Example 6.7 
below). In other words,

$$
T(k):=\left(\begin{array}{c}
\frac{T_{1}}{\vdots} \\
\frac{T_{k}}{T_{k}}
\end{array}\right)
$$

Since $A$ satisfies property (iv), the partition $\mu^{k}$ can be recovered by upward justifying the nonzero entries of $T(k)$. In particular, the entire collection $T_{1}, \ldots, T_{K}$ uniquely determines the matrix $A$.

We now define the "complementary" $L_{k} \times M$ matrix $S_{k}$ by

$$
S_{k}[x, y]:=1-T_{k}[x, M-y+1]
$$

and $S(k)$ as the corresponding column matrix with block entries $S_{1}, \ldots, S_{k}$. It is easy that if the nonzero entries of $S(k)$ are justified upwards, we get the dual partition $\left(\mu^{k}\right)^{*}$ in rectangle $\left(M^{M_{k}}\right)$ where $M_{k}:=\sum_{i=1}^{k} L_{i}$. Hence $S_{1}, \ldots, S_{K}$ determines some matrix $B$ in the same way that $T_{1}, \ldots, T_{K}$ determines $A$. Also note that we can recover $T_{k}$ from $S_{k}$ by applying the complementary operation to $S_{k}$. Theorem 6.5 is a consequence of the following proposition.

Proposition 6.6. The collection $S_{1}, \ldots, S_{K}$ determines a configuration matrix for the sequence $\left(L_{1}, \ldots, L_{K} ; M-N\right)$.

Proof. Let $B=\left[B_{1}|\cdots| B_{K}\right]$ denote the matrix corresponding to the collection $S_{1}, \ldots, S_{K}$. We will show that $B$ is a configuration matrix for the sequence $\left(L_{1}, \ldots, L_{K} ; M-N\right)$. In this case, property (v) is the most challenging to prove. Hence most the argument to dedicated to this part of the proof.

First, note that $B$ trivially satisfies property (i). Next, we observe that $A$ satisfies properties (ii) and (iii) if and only if the matrix $T(K)$ has $M$ columns where each column sum is equal to $N$. Since $S(K)$ has the same number of columns as $T(K)$ with column sums of $M-N$, we get that $B$ also satisfies properties (ii) and (iii). Property (iv) follows from the fact that if we upwards justify the entries of $S(k)$ we get the shape of the dual partition $\left(\mu^{k}\right)^{*}$.

We now prove that $B$ satisfies property (v) by contradiction. Suppose there exists $B_{k}$ and integers $j, l$ such that

$$
\sum_{i=1}^{l}\left(B_{k}[i, j]-B_{k}[i, j+1]\right)<B_{k}[l+1, j+1] .
$$

This implies there exists an integer $l^{\prime}$ such that

$$
\sum_{i=l^{\prime}+1}^{M}\left(S_{k}[j, i]-S_{k}[j+1, i]\right)<0
$$


with

$$
S_{k}\left[j, l^{\prime}+1\right]=0 \quad \text { and } \quad S_{k}\left[j+1, l^{\prime}+1\right]=1 .
$$

Conversely, assume there exists an integer $l^{\prime}$ such that (6-8) and (6-9) are true. By (6-9), there exists an integer $l^{\prime \prime}$ such that

$$
\sum_{i=l^{\prime}+1}^{M} S_{k}[j, i]=\sum_{i=1}^{l^{\prime \prime}} B_{k}[i, j] \quad \text { and } \quad \sum_{i=l^{\prime}+1}^{M} S_{k}[j+1, i] \leq \sum_{i=1}^{l^{\prime \prime}+1} B_{k}[i, j+1] .
$$

Hence by (6-8),

$-B_{k}\left[l^{\prime \prime}+1, j+1\right]+\sum_{i=1}^{l^{\prime \prime}}\left(B_{k}[i, j]-B_{k}[i, j+1]\right) \leq \sum_{i=l^{\prime}+1}^{M}\left(S_{k}[j, i]-S_{k}[j+1, i]\right)<0$.

Observe that if (6-8) is true for $l$, then there is always some integer $l^{\prime} \leq l$ for which both (6-8) and (6-9) are true. Thus the failure of property (v) is equivalent to (6-8). By definition of $S_{k}$ and (6-8), we have

$$
\sum_{i=1}^{M-l^{\prime}}\left(T_{k}[j+1, i]-T_{k}[j, i]\right)<0 .
$$

Since the row sums of $T_{k}$ equal $N$,

$$
\sum_{i=M-l^{\prime}+1}^{M}\left(T_{k}[j, i]-T_{k}[j+1, i]\right)<0
$$

Therefore the matrix $A$ also fails to satisfy property (v) which is a contradiction. This completes the proof.

Example 6.7. Consider $N=4$ and $\mathbf{L}=(2,2,2,1)$ as in Example 6.4. Then $\mu^{4}$, as a union of Littlewood-Richardson skew tableaux, is equal to

\begin{tabular}{|l|l|l|l|l|l|l|}
\hline 1 & 1 & 1 & 1 & 1 & 1 & 1 \\
\hline 2 & 2 & 2 & 2 & 2 & 1 & 1 \\
\hline 1 & 2 & 2 & 1 & 1 & 2 & 2 \\
\hline 2 & 2 & 2 & 1 & 1 & 1 & 1 \\
\hline
\end{tabular}


We have

$$
T(4)=\left(\begin{array}{lllllll}
1 & 1 & 1 & 1 & 0 & 0 & 0 \\
1 & 1 & 1 & 1 & 0 & 0 & 0 \\
\hline 1 & 0 & 0 & 0 & 1 & 1 & 1 \\
1 & 1 & 1 & 0 & 1 & 0 & 0 \\
\hline 0 & 0 & 0 & 1 & 1 & 1 & 1 \\
0 & 1 & 1 & 0 & 0 & 1 & 1 \\
\hline 0 & 0 & 0 & 1 & 1 & 1 & 1
\end{array}\right) \quad S(4)=\left(\begin{array}{lllllll}
1 & 1 & 1 & 0 & 0 & 0 & 0 \\
1 & 1 & 1 & 0 & 0 & 0 & 0 \\
\hline 0 & 0 & 0 & 1 & 1 & 1 & 0 \\
1 & 1 & 0 & 1 & 0 & 0 & 0 \\
\hline 0 & 0 & 0 & 0 & 1 & 1 & 1 \\
0 & 0 & 1 & 1 & 0 & 0 & 1 \\
0 & 0 & 0 & 0 & 1 & 1 & 1
\end{array}\right)
$$

Upward justifying the nonzero entries of $S(4)$ gives the union of LittlewoodRichardson skew tableaux and corresponding configuration matrix

\begin{tabular}{|l|l|l|l|l|l|l|}
\hline 1 & 1 & 1 & 1 & 1 & 1 & 1 \\
\hline 2 & 2 & 2 & 2 & 1 & 1 & 2 \\
\hline 2 & 2 & 2 & 2 & 1 & 1 & 1 \\
\hline
\end{tabular}

$$
B=\left(\begin{array}{ll|ll|ll|l}
3 & 0 & 3 & 0 & 1 & 0 & 0 \\
0 & 3 & 0 & 1 & 2 & 1 & 0 \\
0 & 0 & 0 & 2 & 0 & 2 & 3
\end{array}\right),
$$

and hence $(2,2,2,1) \in \operatorname{TFF}(7 / 3,3)$.

\section{Examples and tables of TFF sequences}

This section is divided into two parts. In the first part we give several examples of existence of tight fusion frames using skew Littlewood-Richardson tableaux as in Example 4.5. In the second part, we give a complete list of tight fusion frame sequences for $N \leq 9$ and $\alpha \leq 2$ by listing all maximal elements in the partial order induced by majorization.

Examples of skew Littlewood-Richardson tableaux. The following are some examples of Littlewood-Richardson tableaux in the cases of $N=3,5,7$. Readers who are interested in combinatorial spatial and Naimark duality as discussed in Section 6 are encouraged to apply the bijective constructions to these examples.

$N=3$ and $\mathbf{L}=(3,2,1), \mathbf{L}=(2,1,1,1)$, and $\mathbf{L}=(1,1,1,1)$ :

\begin{tabular}{|l|l|l|l|l|l|}
\hline 1 & 1 & 1 & 1 & 1 & 1 \\
\hline 2 & 2 & 2 & 2 & 2 & 2 \\
\hline 3 & 3 & 3 & 1 & 1 & 1 \\
\hline
\end{tabular}

\begin{tabular}{|l|l|l|l|l|}
\hline 1 & 1 & 1 & 1 & 1 \\
\hline 2 & 2 & 2 & 1 & 1 \\
\hline 1 & 1 & 1 & 1 & 1 \\
\hline
\end{tabular}

\begin{tabular}{|l|l|l|l|}
\hline 1 & 1 & 1 & 1 \\
\hline 1 & 1 & 1 & 1 \\
\hline 1 & 1 & 1 & 1 \\
\hline
\end{tabular}

$N=5$ and $\mathbf{L}=(2,2,2,2)$ and $\mathbf{L}=(3,3,3,3)$ :

\begin{tabular}{|l|l|l|l|l|l|l|l|}
\hline 1 & 1 & 1 & 1 & 1 & 1 & 1 & 1 \\
\hline 2 & 2 & 2 & 2 & 2 & 2 & 1 & 1 \\
\hline 1 & 1 & 2 & 2 & 1 & 1 & 2 & 2 \\
\hline 2 & 2 & 1 & 1 & 1 & 1 & 1 & 1 \\
\hline 2 & 2 & 2 & 2 & 2 & 2 & 2 & 2 \\
\hline
\end{tabular}

\begin{tabular}{|l|l|l|l|l|l|l|l|l|l|l|l|}
\hline 1 & 1 & 1 & 1 & 1 & 1 & 1 & 1 & 1 & 1 & 1 & 1 \\
\hline 2 & 2 & 2 & 2 & 2 & 2 & 2 & 2 & 1 & 1 & 2 & 2 \\
\hline 3 & 3 & 3 & 3 & 3 & 3 & 1 & 1 & 1 & 1 & 1 & 1 \\
\hline 2 & 2 & 3 & 3 & 2 & 2 & 2 & 2 & 2 & 2 & 2 & 2 \\
\hline 3 & 3 & 3 & 3 & 3 & 3 & 3 & 3 & 3 & 3 & 3 & 3 \\
\hline
\end{tabular}


$N=7$ and $\mathbf{L}=(4,3,3,1,1)$ and $\mathbf{L}=(3,2,2,2,1)$ :

\begin{tabular}{|l|l|l|l|l|l|l|l|l|l|l|l|}
\hline 1 & 1 & 1 & 1 & 1 & 1 & 1 & 1 & 1 & 1 & 1 & 1 \\
\hline 2 & 2 & 2 & 2 & 2 & 2 & 2 & 2 & 2 & 2 & 2 & 2 \\
\hline 3 & 3 & 3 & 3 & 3 & 3 & 3 & 3 & 3 & 1 & 1 & 1 \\
\hline 4 & 4 & 4 & 4 & 4 & 4 & 4 & 1 & 1 & 2 & 2 & 2 \\
\hline 1 & 1 & 3 & 3 & 3 & 1 & 1 & 2 & 2 & 3 & 3 & 3 \\
\hline 2 & 2 & 2 & 2 & 3 & 3 & 1 & 1 & 1 & 1 & 1 & 1 \\
\hline 3 & 3 & 3 & 3 & 1 & 1 & 1 & 1 & 1 & 1 & 1 & 1 \\
\hline
\end{tabular}

\begin{tabular}{|l|l|l|l|l|l|l|l|l|l|}
\hline 1 & 1 & 1 & 1 & 1 & 1 & 1 & 1 & 1 & 1 \\
\hline 2 & 2 & 2 & 2 & 2 & 2 & 2 & 2 & 2 & 2 \\
\hline 3 & 3 & 3 & 3 & 3 & 3 & 3 & 1 & 1 & 1 \\
\hline 1 & 1 & 1 & 1 & 1 & 1 & 1 & 2 & 2 & 2 \\
\hline 2 & 2 & 2 & 2 & 2 & 1 & 1 & 1 & 1 & 1 \\
\hline 1 & 2 & 2 & 1 & 1 & 2 & 2 & 2 & 2 & 2 \\
\hline 2 & 2 & 2 & 1 & 1 & 1 & 1 & 1 & 1 & 1 \\
\hline
\end{tabular}

Tables of maximal tight fusion frames. At the end of this section we give a complete list of tight fusion frames for $N \leq 9$ and $\alpha \leq 2$ by listing all maximal elements in the partial order induced by majorization. These lists are generated by applying the techniques developed in this paper. In particular, we use the following methods

- Constructing Littlewood-Richardson tableaux as in Corollary 4.4.

- Recursive construction using spatial and Naimark duality.

- Recursive construction using Lemma 7.1.

- Applying inequalities of Theorem 3.3 or 5.4.

The following lemma follows from the Naimark duality.

Lemma 7.1. Assume that $L_{1}=N(\alpha-1)$. Then, $\mathbf{L} \in \operatorname{TFF}(\alpha, N)$ if and only if $\mathbf{L}^{\prime} \in \operatorname{TFF}(\tilde{\alpha}-1, N(\alpha-1))$ where $\mathbf{L}^{\prime}=\left(L_{2} \geq \cdots \geq L_{k}\right)$ and $1 / \alpha+1 / \tilde{\alpha}=1$.

It is easy to see that maximality under the majorization partial order is preserved under these dualities and the lemma above. Unfortunately, there are several TFF sequences missed by majorization and the recursive generation techniques mentioned above. These sequences were only found by brute force construction of Littlewood-Richardson tableaux. The first maximal tight fusion frame sequence missed by recursion is $(4,2,2,2,1)$ where $N=6$. Hence, it might be of interest to illustrate how to construct a tight fusion frame for this sequence.

Example 7.2. Let $N=6$ and $\mathbf{L}=(4,2,2,2,1)$. The first step in our construction is identifying a skew Littlewood-Richardson tableaux corresponding to our TFF sequence.

\begin{tabular}{|l|l|l|l|l|l|l|l|l|l|l|}
\hline 1 & 1 & 1 & 1 & 1 & 1 & 1 & 1 & 1 & 1 & 1 \\
\hline 2 & 2 & 2 & 2 & 2 & 2 & 2 & 2 & 2 & 1 & 1 \\
\hline 3 & 3 & 3 & 3 & 3 & 3 & 1 & 1 & 1 & 2 & 2 \\
\hline 4 & 4 & 4 & 4 & 4 & 4 & 1 & 1 & 1 & 1 & 1 \\
\hline 1 & 2 & 2 & 1 & 2 & 1 & 2 & 2 & 2 & 2 & 2 \\
\hline 2 & 2 & 2 & 2 & 2 & 1 & 1 & 1 & 1 & 1 & 1 \\
\hline
\end{tabular}


The above tableaux shows the existence of projections $P_{1}, \ldots, P_{5}$ in $\mathbb{R}^{6}$ with (7-1) $\sum_{i=1}^{5} P_{i}=\frac{11}{6} \mathbf{I}, \quad \operatorname{rank} P_{1}=4, \operatorname{rank} P_{2}=\operatorname{rank} P_{3}=\operatorname{rank} P_{4}=2, \operatorname{rank} P_{5}=1$.

By Corollary 4.6, the tableaux also contains information on the eigenvalues of the intermediate partial sums of projections in (7-1).

\begin{tabular}{cc}
\hline sum of projections & eigenvalue list \\
\hline$P_{1}$ & $(1,1,1,1,0,0)$ \\
$P_{1}+P_{2}$ & $\left(\frac{11}{6}, \frac{9}{6}, 1,1, \frac{3}{6}, \frac{1}{6}\right)$ \\
$P_{1}+P_{2}+P_{3}$ & $\left(\frac{11}{6}, \frac{11}{6}, \frac{11}{6}, 1, \frac{5}{6}, \frac{4}{6}\right)$ \\
$P_{1}+\cdots+P_{4}$ & $\left(\frac{11}{6}, \frac{11}{6}, \frac{11}{6}, \frac{11}{6}, \frac{11}{6}, \frac{5}{6}\right)$ \\
$P_{1}+\cdots+P_{5}$ & $\left(\frac{11}{6}, \frac{11}{6}, \frac{11}{6}, \frac{11}{6}, \frac{11}{6}, \frac{11}{6}\right)$ \\
\hline
\end{tabular}

Equipped with this information and a symbolic computation program such as Mathematica we can construct an explicit tight fusion frame in $\mathbb{R}^{6}$ associated with the sequence $(4,2,2,2,1)$. The matrix below shows an orthonormal basis (column) vectors for the corresponding ranges of projections $P_{i}, i=1, \ldots, 5$.

$$
\left(\begin{array}{cccc|cc|cc|cc|c}
1 & 0 & 0 & 0 & \frac{5}{6} & 0 & -\sqrt{\frac{5}{72}} & 0 & \sqrt{\frac{5}{72}} & 0 & 0 \\
0 & 1 & 0 & 0 & 0 & \frac{1}{2} & -\frac{1}{2 \sqrt{2}} & -\frac{1}{3} & -\frac{1}{2 \sqrt{2}} & \frac{1}{3} & \frac{1}{3} \\
0 & 0 & 1 & 0 & 0 & 0 & 0 & \frac{\sqrt{5}}{3} & 0 & \frac{\sqrt{5}}{6} & \frac{\sqrt{5}}{6} \\
0 & 0 & 0 & 1 & 0 & 0 & 0 & 0 & 0 & \sqrt{\frac{5}{12}} & -\sqrt{\frac{5}{12}} \\
0 & 0 & 0 & 0 & 0 & -\frac{\sqrt{3}}{2} & \frac{1}{2 \sqrt{6}} & -\frac{1}{\sqrt{3}} & \frac{1}{2 \sqrt{6}} & \frac{1}{\sqrt{3}} & \frac{1}{\sqrt{3}} \\
0 & 0 & 0 & 0 & -\frac{\sqrt{11}}{6} & 0 & -\sqrt{\frac{55}{72}} & 0 & \sqrt{\frac{55}{72}} & 0 & 0
\end{array}\right)
$$

A direct calculation shows that: (i) columns are orthonormal to each other in every block, and (ii) rows are orthogonal with norms $\sqrt{11 / 6}$. This proves the existence of a TFF (7-1).

It is worth noting that the Example 7.2 can not be obtained using the spectral tetris construction (STC). The STC has been recently introduced by Casazza et al. [2012] who gave an algorithmic way of constructing sparse fusion frames. Among other things, the authors have shown that the ranks $\mathbf{L}$ of spectral tetris fusion frames must necessarily satisfy $\mathbf{L} \preccurlyeq \mathbf{L}^{\prime}$, where $\mathbf{L}^{\prime}$ is a sequence of ranks of the reference fusion frame. 
Moreover, in the tight case this condition is also sufficient, and hence [Casazza et al. 2012, Theorem 3.3] characterizes possible ranks obtained by the STC in the case when the frame bound $\alpha \geq 2$. Combining this with Naimark complements, see Theorem 2.6, this yields TFFs also in the case $1<\alpha<2$. In particular, we have $\operatorname{TFF}(11 / 6,6)=\operatorname{TFF}(11 / 5,5)$. A direct calculation of the reference fusion frame corresponding to eigenvalues $(11 / 5,11 / 5,11 / 5,11 / 5,11 / 5)$ yields a TFF sequence $(3,3,3,2)$. This happens to be another maximal element of $\operatorname{TFF}(11 / 6,6)$ which is not comparable with $(4,2,2,2,1)$ with respect to the majorization relation $\preccurlyeq$. Hence, the above example can not be obtained by the STC even when paired with Naimark duality.

List of maximal TFF sequences for $N \leq 9$ and $\alpha \leq 2$.

\begin{tabular}{|c|c|}
\hline \multicolumn{2}{|c|}{$N=3$} \\
\hline$\alpha$ & max elements \\
\hline 1 & $(3)$ \\
$4 / 3$ & $(1,1,1,1)$ \\
$5 / 3$ & $(2,1,1,1)$ \\
2 & $(3,3)$ \\
\hline
\end{tabular}

\begin{tabular}{|c|c|}
\hline \multicolumn{2}{|c|}{$N=4$} \\
\hline$\alpha$ & max elements \\
\hline 1 & $(4)$ \\
$5 / 4$ & $(1,1,1,1,1)$ \\
$6 / 4$ & $(2,2,2)$ \\
$7 / 4$ & $(3,1,1,1,1),(2,2,2,1)$ \\
2 & $(4,4)$ \\
\hline
\end{tabular}

\begin{tabular}{|c|c|}
\hline \multicolumn{2}{|c|}{$N=5$} \\
\hline$\alpha$ & max elements \\
\hline 1 & $(5)$ \\
$6 / 5$ & $(1,1,1,1,1,1)$ \\
$7 / 5$ & $(2,2,1,1,1)$ \\
$8 / 5$ & $(3,2,1,1,1),(2,2,2,2)$ \\
$9 / 5$ & $(4,1,1,1,1,1),(3,2,2,2)$ \\
2 & $(5,5)$ \\
\hline
\end{tabular}

\begin{tabular}{|c|c|}
\hline \multicolumn{2}{|c|}{$N=6$} \\
\hline$\alpha$ & max elements \\
\hline 1 & $(6)$ \\
$7 / 6$ & $(1,1,1,1,1,1,1)$ \\
$8 / 6$ & $(2,2,2,2)$ \\
$9 / 6$ & $(3,3,3)$ \\
$10 / 6$ & $(4,2,2,2)$ \\
$11 / 6$ & $(5,1,1,1,1,1,1),(4,2,2,2,1),(3,3,3,2)$ \\
2 & $(6,6)$ \\
\hline
\end{tabular}




\begin{tabular}{|c|c|}
\hline \multicolumn{2}{|c|}{$N=7$} \\
\hline$\alpha$ & max elements \\
\hline 1 & $(7)$ \\
$8 / 7$ & $(1,1,1,1,1,1,1,1)$ \\
$9 / 7$ & $(2,2,2,1,1,1)$ \\
$10 / 7$ & $(3,3,1,1,1,1),(3,2,2,2,1)$ \\
$11 / 7$ & $(4,3,1,1,1,1),(4,2,2,2,1)$ \\
$12 / 7$ & $(5,2,2,1,1,1),(4,3,3,1,1),(3,3,3,3)$ \\
$13 / 7$ & $(6,1,1,1,1,1,1,1),(5,2,2,2,2),(4,3,3,3)$ \\
2 & $(7,7)$ \\
\hline
\end{tabular}

\begin{tabular}{|c|c|}
\hline \multicolumn{2}{|c|}{$N=8$} \\
\hline$\alpha$ & max elements \\
\hline 1 & $(8)$ \\
$9 / 8$ & $(1,1,1,1,1,1,1,1,1)$ \\
$10 / 8$ & $(2,2,2,2,2)$ \\
$11 / 8$ & $(3,2,2,2,2),(3,3,2,1,1,1)$ \\
$12 / 8$ & $(4,4,4)$ \\
$13 / 8$ & $(5,3,2,1,1,1),(5,2,2,2,2),(4,4,2,2,1)$ \\
$14 / 8$ & $(6,2,2,2,2),(5,3,3,2,1),(4,4,4,2)$ \\
$15 / 8$ & $(7,1,1,1,1,1,1,1),(6,2,2,2,2,1),(5,3,3,2,2),(4,4,4,3)$ \\
2 & $(8,8)$ \\
\hline
\end{tabular}

\begin{tabular}{|c|c|}
\hline \multicolumn{2}{|c|}{$N=9$} \\
\hline$\alpha$ & max elements \\
\hline 1 & $(9)$ \\
$10 / 9$ & $(1,1,1,1,1,1,1,1,1,1)$ \\
$11 / 9$ & $(2,2,2,2,1,1,1)$ \\
$12 / 9$ & $(3,3,3,3)$ \\
$13 / 9$ & $(4,4,1,1,1,1,1),(4,3,2,2,2),(3,3,3,3,1)$ \\
$14 / 9$ & $(5,4,1,1,1,1,1),(5,3,2,2,2),(4,3,3,3,1)$ \\
$15 / 9$ & $(6,3,3,3)$ \\
$16 / 9$ & $(7,2,2,2,1,1,1),(6,3,3,3,1),(5,4,4,2,1),(4,4,4,4)$ \\
$17 / 9$ & $(8,1,1,1,1,1,1,1,1,1),(7,2,2,2,2,2),(6,3,3,3,2),(5,4,4,4)$ \\
2 & $(9,9)$ \\
\hline
\end{tabular}

The following sequences are missed by the recursive construction of spatial and Naimark duality and Lemma 7.1. These sequences were obtained applying Theorem 3.3 and constructing Littlewood-Richardson tableaux. 


\begin{tabular}{|c|c|}
\hline$N$ & TFF sequences \\
\hline 6 & $(4,2,2,2,1)$ \\
7 & $(4,3,3,1,1),(5,2,2,2,2)$ \\
8 & $(5,3,3,2,1),(6,2,2,2,2,1),(5,3,3,2,2)$ \\
9 & $(6,3,3,3,1),(5,4,4,2,1),(7,2,2,2,2,2),(6,3,3,3,2),(5,4,4,4)$ \\
\hline
\end{tabular}

We also remark that the sequences $(4,4,2,2,1)$ for $N=8$ and $(4,3,3,3,1)$ for $N=9$ can be recursively found from the "missed" sequences $(4,3,3,1,1)$ for $N=7$ and $(4,2,2,2,1)$ for $N=6$, respectively. Finally, among the eleven maximal TFF sequences listed above only the sequence $(5,4,4,4)$ for $N=9$ can be obtained using the spectral tetris construction (STC) paired with the Naimark duality. A construction of explicit tight fusion frames corresponding to the remaining ten maximal TFF sequences requires a procedure similar to Example 7.2.

\section{Acknowledgments}

Authors would like to thank Stephanie van Willigenburg for useful discussions. Bownik was partially supported by NSF grant DMS-1265711. Luoto and Richmond were partially supported by the Natural Sciences and Engineering Research Council of Canada.

\section{References}

[Andrews 1976] G. E. Andrews, The theory of partitions, Encyclopedia of Mathematics and its Applications 2, Addison-Wesley, Reading, MA, 1976. MR 58 \#27738 Zbl 0371.10001

[Antezana et al. 2007] J. Antezana, P. G. Massey, M. A. Ruiz, and D. Stojanoff, "The Schur-Horn theorem for operators and frames with prescribed norms and frame operator", Illinois J. Math. 51:2 (2007), 537-560. MR 2009g:42049 Zbl 1137.42008

[Bodmann 2007] B. G. Bodmann, "Optimal linear transmission by loss-insensitive packet encoding”, Appl. Comput. Harmon. Anal. 22:3 (2007), 274-285. MR 2008c:42031 Zbl 1193.42113

[Boufounos et al. 2011] P. Boufounos, G. Kutyniok, and H. Rauhut, "Sparse recovery from combined fusion frame measurements”, IEEE Trans. Inform. Theory 57:6 (2011), 3864-3876. MR 2012e:94032

[Calderbank et al. 2011] R. Calderbank, P. G. Casazza, A. Heinecke, G. Kutyniok, and A. Pezeshki, "Sparse fusion frames: existence and construction", Adv. Comput. Math. 35:1(2011), 1-31. MR 2012d: 42067 Zbl 1264.94042

[Casazza and Fickus 2009] P. G. Casazza and M. Fickus, "Minimizing fusion frame potential", Acta Appl. Math. 107:1-3 (2009), 7-24. MR 2010e:42042 Zbl 1175.42010

[Casazza and Kutyniok 2004] P. G. Casazza and G. Kutyniok, "Frames of subspaces", pp. 87-113 in Wavelets, frames and operator theory (College Park, MD, 2003), edited by C. Heil et al., Contemporary Mathematics 345, American Mathematical Society, Providence, RI, 2004. MR 2005e:42090 Zbl 1058.42019

[Casazza and Kutyniok 2013] P. G. Casazza and G. Kutyniok, "Fusion frames", pp. 437-477 in Finite frames: theory and applications, edited by P. G. Casazza and G. Kutyniok, Birkhäuser, New York, 2013. MR 2964018 Zbl 1261.42048 
[Casazza et al. 2008] P. G. Casazza, G. Kutyniok, and S. Li, "Fusion frames and distributed processing”, Appl. Comput. Harmon. Anal. 25:1 (2008), 114-132. MR 2009d:42094 Zbl 1258.42029

[Casazza et al. 2011] P. G. Casazza, M. Fickus, D. G. Mixon, Y. Wang, and Z. Zhou, "Constructing tight fusion frames", Appl. Comput. Harmon. Anal. 30:2 (2011), 175-187. MR 2012c:42069 Zbl 1221.42052

[Casazza et al. 2012] P. G. Casazza, M. Fickus, A. Heinecke, Y. Wang, and Z. Zhou, "Spectral tetris fusion frame constructions", J. Fourier Anal. Appl. 18:4 (2012), 828-851. MR 2984371 Zbl 1257.42042

[Chebira et al. 2011] A. Chebira, M. Fickus, and D. G. Mixon, "Filter bank fusion frames", IEEE Trans. Signal Process. 59:3 (2011), 953-963. MR 2012d:94015

[Fillmore 1969] P. A. Fillmore, “On sums of projections”, J. Funct. Anal. 4:1 (1969), 146-152. MR 39 \#7455 Zbl 0176.43404

[Fong and Murphy 1985] C. K. Fong and G. J. Murphy, "Averages of projections", J. Operator Theory 13:2 (1985), 219-225. MR 86h:47071 Zbl 0614.47011

[Fulton 1997] W. Fulton, Young tableaux: with applications to representation theory and geometry, London Mathematical Society Student Texts 35, Cambridge University Press, 1997. MR 99f:05119 Zbl 0878.14034

[Fulton 2000] W. Fulton, "Eigenvalues, invariant factors, highest weights, and Schubert calculus", Bull. Amer. Math. Soc. (N.S.) 37:3 (2000), 209-249. MR 2001g:15023 Zbl 0994.15021

[Halpern et al. 2013] H. Halpern, V. Kaftal, P. W. Ng, and S. Zhang, "Finite sums of projections in von Neumann algebras", Trans. Amer. Math. Soc. 365:5 (2013), 2409-2445. MR 3020103 Zbl 1275.47088

[Horn 1962] A. Horn, "Eigenvalues of sums of Hermitian matrices", Pacific J. Math. 12 (1962), 225-241. MR 25 \#3941 Zbl 0112.01501

[Kaftal and Weiss 2010] V. Kaftal and G. Weiss, "An infinite dimensional Schur-Horn theorem and majorization theory", J. Funct. Anal. 259:12 (2010), 3115-3162. MR 2011k:47030 Zbl 1202.15035

[Kaftal et al. 2011] V. Kaftal, P. W. Ng, and S. Zhang, "Positive combinations and sums of projections in purely infinite simple $\mathbb{C}^{*}$-algebras and their multiplier algebras", Proc. Amer. Math. Soc. 139:8 (2011), 2735-2746. MR 2012f:46100 Zbl 1230.46049

[Klyachko 1998] A. A. Klyachko, "Stable bundles, representation theory and Hermitian operators", Selecta Math. (N.S.) 4:3 (1998), 419-445. MR 2000b:14054 Zbl 0915.14010

[Knutson and Tao 1999] A. Knutson and T. Tao, "The honeycomb model of $\mathrm{GL}_{n}(\mathbb{C})$ tensor products, I: Proof of the saturation conjecture", J. Amer. Math. Soc. 12:4 (1999), 1055-1090. MR 2000c:20066 Zbl 0944.05097

[Knutson and Tao 2001] A. Knutson and T. Tao, "Honeycombs and sums of Hermitian matrices", Notices Amer. Math. Soc. 48:2 (2001), 175-186. MR 2002g:15020 Zbl 1047.15006

[Knutson et al. 2004] A. Knutson, T. Tao, and C. Woodward, "The honeycomb model of GL $(\mathbb{C})$ tensor products, II: Puzzles determine facets of the Littlewood-Richardson cone", J. Amer. Math. Soc. 17:1 (2004), 19-48. MR 2005f:14105 Zbl 1043.05111

[Kruglyak et al. 2002] S. A. Kruglyak, V. I. Rabanovich, and Y. S. Samoŭlenko, "O суммах проекторов", Funkts. Anal. Prilozh. 36:3 (2002), 20-35. Translated as "On sums of projections" in Funct. Anal. Appl. 36:3 (2002), 182-195. MR 2004e:47021 Zbl 1038.47001

[Kruglyak et al. 2003] S. A. Kruglyak, V. I. Rabanovich, and Y. S. Samoŭlenko, "Decomposition of a scalar matrix into a sum of orthogonal projections", Linear Algebra Appl. 370 (2003), 217-225. MR 2004f:15045 Zbl 1028.15010 
[Kutyniok et al. 2009] G. Kutyniok, A. Pezeshki, R. Calderbank, and T. Liu, "Robust dimension reduction, fusion frames, and Grassmannian packings", Appl. Comput. Harmon. Anal. 26:1 (2009), 64-76. MR 2010a:94023 Zbl 1283.42046

[Massey et al. 2010] P. G. Massey, M. A. Ruiz, and D. Stojanoff, "The structure of minimizers of the frame potential on fusion frames", J. Fourier Anal. Appl. 16:4 (2010), 514-543. MR 2011f:42038 Zbl 1194.42039

[Okada 1998] S. Okada, "Applications of minor summation formulas to rectangular-shaped representations of classical groups", J. Algebra 205:2 (1998), 337-367. MR 99g:20081 Zbl 0915.20023

Received October 7, 2013. Revised September 27, 2014.

MARCIN BOWNIK

DEPARTMENT OF MATHEMATICS

UNIVERSITY OF OREGON

EUGENE, OR 97403

UNITED STATES

mbownik@uoregon.edu

KURT LUOTO

DEPARTMENT OF MATHEMATICS

UNIVERSITY OF BRITISH COLUMBIA

VANCOUVER BC V6T 1 Z2

CANADA

kwluoto@math.ubc.ca

EDWARD RICHMOND

DEPARTMENT OF MATHEMATICS

OKLAHOMA STATE UNIVERSITY

STILLWATER, OK 74078

UNITED STATES

edward.richmond@okstate.edu 


\title{
PACIFIC JOURNAL OF MATHEMATICS
}

\author{
msp.org/pjm
}

Founded in 1951 by E. F. Beckenbach (1906-1982) and F. Wolf (1904-1989)

\section{EDITORS}

Don Blasius (Managing Editor)

Department of Mathematics

University of California

Los Angeles, CA 90095-1555

blasius@math.ucla.edu

\author{
Paul Balmer \\ Department of Mathematics \\ University of California \\ Los Angeles, CA 90095-1555 \\ balmer@math.ucla.edu \\ Robert Finn \\ Department of Mathematics \\ Stanford University \\ Stanford, CA 94305-2125 \\ finn@math.stanford.edu \\ Sorin Popa \\ Department of Mathematics \\ University of California \\ Los Angeles, CA 90095-1555 \\ popa@math.ucla.edu
}

\author{
Vyjayanthi Chari \\ Department of Mathematics \\ University of California \\ Riverside, CA 92521-0135 \\ chari@math.ucr.edu \\ Kefeng Liu \\ Department of Mathematics \\ University of California \\ Los Angeles, CA 90095-1555 \\ liu@math.ucla.edu \\ Jie Qing \\ Department of Mathematics \\ University of California \\ Santa Cruz, CA 95064 \\ qing@ cats.ucsc.edu
}

\section{PRODUCTION}

Silvio Levy, Scientific Editor, production@msp.org

\section{SUPPORTING INSTITUTIONS}

ACADEMIA SINICA, TAIPEI

CALIFORNIA INST. OF TECHNOLOGY

INST. DE MATEMÁTICA PURA E APLICADA

KEIO UNIVERSITY

MATH. SCIENCES RESEARCH INSTITUTE

NEW MEXICO STATE UNIV.

OREGON STATE UNIV.

\author{
STANFORD UNIVERSITY \\ UNIV. OF BRITISH COLUMBIA \\ UNIV. OF CALIFORNIA, BERKELEY \\ UNIV. OF CALIFORNIA, DAVIS \\ UNIV. OF CALIFORNIA, LOS ANGELES \\ UNIV. OF CALIFORNIA, RIVERSIDE \\ UNIV. OF CALIFORNIA, SAN DIEGO \\ UNIV. OF CALIF., SANTA BARBARA
}

\author{
Daryl Cooper \\ Department of Mathematics \\ University of California \\ Santa Barbara, CA 93106-3080 \\ cooper@math.ucsb.edu \\ Jiang-Hua Lu \\ Department of Mathematics \\ The University of Hong Kong \\ Pokfulam Rd., Hong Kong \\ jhlu@maths.hku.hk \\ Paul Yang \\ Department of Mathematics \\ Princeton University \\ Princeton NJ 08544-1000 \\ yang@math.princeton.edu
}

These supporting institutions contribute to the cost of publication of this Journal, but they are not owners or publishers and have no responsibility for its contents or policies.

See inside back cover or msp.org/pjm for submission instructions.

The subscription price for 2015 is US \$420/year for the electronic version, and \$570/year for print and electronic.

Subscriptions, requests for back issues and changes of subscribers address should be sent to Pacific Journal of Mathematics, P.O. Box 4163, Berkeley, CA 94704-0163, U.S.A. The Pacific Journal of Mathematics is indexed by Mathematical Reviews, Zentralblatt MATH, PASCAL CNRS Index, Referativnyi Zhurnal, Current Mathematical Publications and Web of Knowledge (Science Citation Index).

The Pacific Journal of Mathematics (ISSN 0030-8730) at the University of California, c/o Department of Mathematics, 798 Evans Hall \#3840, Berkeley, CA 94720-3840, is published twelve times a year. Periodical rate postage paid at Berkeley, CA 94704, and additional mailing offices. POSTMASTER: send address changes to Pacific Journal of Mathematics, P.O. Box 4163, Berkeley, CA 94704-0163.

PJM peer review and production are managed by EditFLOW ${ }^{\circledR}$ from Mathematical Sciences Publishers.

\section{PUBLISHED BY}

\section{mathematical sciences publishers \\ nonprofit scientific publishing}

http://msp.org/

(C) 2015 Mathematical Sciences Publishers 


\section{PACIFIC JOURNAL OF MATHEMATICS}

Volume $275 \quad$ No. $2 \quad$ June 2015

A combinatorial characterization of tight fusion frames

MARCIN BOWNIK, KURT LUOTO and EDWARD RICHMOND

Combinatorics of finite abelian groups and Weil representations

295

KUnAL DUTTA and AMritANSHU PRASAD

Compact anti-de Sitter 3-manifolds and folded hyperbolic structures on

325 surfaces

FRANÇOIS GUÉRITAUd, FANNY KASSEL and MAXIME WOLFF

Circular handle decompositions of free genus one knots

361

FABIOLA MANJARREZ-GUTIÉRREZ, VÍCTOR NÚÑEZ and

ENRIQUE RAMÍREZ-LOSADA

A pointwise a-priori estimate for the $\bar{\partial}$-Neumann problem on weakly 409 pseudoconvex domains

R. MichaEL RANGE

Explicit Hilbert-Kunz functions of $2 \times 2$ determinantal rings

MARCUS ROBINSON and IRENA SWANSON

The Johnson-Morita theory for the ring of Fricke characters of free groups

\section{TAKAO SATOH}

Global representations of the conformal group and eigenspaces of the Yamabe operator on $S^{1} \times S^{n}$

MARK R. SEPANSKI and Jose A. Franco

Rota-Baxter operators on the polynomial algebra, integration, and averaging operators

Shanghua Zheng, Li GuO and MARKus Rosenkranz

Correction to the article Quiver grassmannians, quiver varieties and the preprojective algebra

Alistair Savage and Peter Tingley 